\title{
Measuring the Development of Airline Networks: Comprehensive Indicators
}

\author{
Chantal Roucolle ${ }^{1}$, Tatiana Seregina ${ }^{2}$, Miguel Urdanoz ${ }^{3}$
}

\begin{abstract}
The literature on airlines presents few studies analyzing the airlines network evolution. We believe that this gap is due to the difficulty of capturing the network complexity in a simple manner. This paper proposes new simple and continuous indicators to measure the airlines' network structure. The methodology to build them is based on graph theory and principal component analysis. We apply this approach to the US domestic market for 2005-2018, and obtain three network indicators. The first one measures how close the network is to a single-center structure. The second indicator measures the airline's ability to provide alternative routes. The third indicator captures the network size. We analyze the indicators evolution across time and show their robustness under different scenarios.
\end{abstract}

Keywords: Airline; Graph theory; Network; Principal Component Analysis (PCA); Indicators

Funding: This research was possible thanks to the financial support of the Occitanie Regional Council.

\section{Highlights:}

- Propose a methodology to build airline network structure indicators

- Combine graph theory and principal component analysis

- Obtain three indicators to characterize airline network structure for US domestic market

- Represent the indicators evolution

- Analyze the impact of the main US mergers on the network structure

\footnotetext{
${ }^{1}$ Chantal Roucolle, École Nationale de l'Aviation Civile - Université de Toulouse, 7 avenue Edouard Belin CS 54005, 31055 Toulouse Cedex 4, France. chantal.latge-roucolle@enac.fr

${ }^{2}$ Corresponding author. Tatiana Seregina, École Nationale de l'Aviation Civile - Université de Toulouse, 7 avenue Edouard Belin CS 54005, 31055 Toulouse Cedex 4, France, and Toulouse Business School - Université Toulouse I, 1 Place Alphonse Jourdain CS 66810, 31068 Toulouse Cedex 7, France. tatiana.seregina@enac.fr

${ }^{3}$ Miguel Urdanoz. Toulouse Business School - Université Toulouse I, 1 Place Alphonse Jourdain CS 66810, 31068 Toulouse Cedex 7, France. m.urdanoz@ @tbs-education.fr , Tel: +33561294743
} 


\section{Introduction}

The air passenger industry is extremely complex and dynamic, and continues to grow rapidly. Since 1970, worldwide air traffic has doubled every 15 years, and according to Airbus forecasts (2017) this growth rate will persist over the next 20 years. In this context, each air carrier adapts its network structure, i.e., airports served and flight schedules. This involves the choice of aircraft type, frequency and finally, number of assigned seats on each flight leg. Scheduled flights form a complex network of connected cities whose organization depends on airlines' strategies of expansion. This study applies a methodology based on graph theory and principal component analysis to build new indicators that reflect an airline's individual network structure. The new indicators allow to draw an innovative map of airline networks.

The evolution of airline networks has been a central concern in the airline literature since the emergence of the first hubs ${ }^{4}$ in the U.S. in the 1970s. The hub-and-spoke network seemed at that time to be a more profitable structure due to economies of density and scope (Caves, Christensen, and Tretheway 1984; Brueckner and Spiller 1994; Nero 1999). Therefore, airlines were expected to implement this type of structure while increasing their size.

However, new operators in the industry that entered the market after the deregulation, namely low-cost carriers (LCCs), adopted a totally different network organization, with highly connected or point-to-point networks. Their network organization together with an offensive pricing strategy and a cost reducing business model allowed them in some cases to achieve higher profits than the legacy carriers. The attention of several authors focused on comparing these two distinct network structures, point-to-point versus huband-spoke, as in Brueckner (2004), Alderighi et al.(2005), Barla and Constantatos (2005), Flores-Fillol (2009), and Silva, Verhoef, and Van den Berg (2014). In most of the cases, perfect hub-and-spoke networks are compared with fully-connected networks. However, reality is more complex and airline network organizations often lies between these two extreme cases. Wojahn (2001) shows that a network combining hub-and-spoke and fully-connected structures could be optimal and that multi-hub networks are not cost minimizing for airlines. Besides, hubbing is only one of several possible network dimensions and is not necessarily the only decision for airlines in terms of network structure. To our knowledge, there have been no studies attempting to analyze the evolution of airline networks from an economic perspective. We believe that this gap is due to the difficulty of capturing the network complexity in a simple manner. The large number of interconnected routes, and the diversity of relationships between those routes, impede constructing an appropriate model and thereby analyze the structural and dynamical properties of the networks.

In this study, we build continuous indicators of airline network structures combining graph theory measures with a principal component analysis. Building continuous indicators has a double purpose. First, it allows to compare and classify airlines according to their network structure and to study the airline's network complex evolution in a simple manner. Second, it allows to study the impact of the network structure over profits, costs or delays. Both purposes are relevant to determine the optimal airline strategies and in consequence the optimal regulation. This article considers the first objective while the second one is left for future research.

To begin with, we consider airlines' networks as graphs and we select graph theory measures relevant to describe the network structure according to the literature on airline networks. Given the large number of graph measures, we propose to apply a principal component analysis (PCA) to synthesize the information they convey. Using PCA we obtain straightforward continuous indicators, the principal components, to characterize the airline networks. We apply this methodology to the airlines operating on the US domestic market from 2005 to 2018 . We show that three principal components reflect most of the information contained in the selected graph measures. These three components allow characterizing the network structure and help to represent its evolution. The first component that we denote as NetCenter, measures the presence of central cities, central in terms of route concentration. The second component, denoted as

\footnotetext{
${ }^{4}$ The literature considers as hubs the airports where one or several airlines propose a large number of flights, and passengers can transfer between flights to get to their intended destination.
} 
NetWeave, represents the airline's ability to mitigate disturbances by providing alternative flights within its network. NetWeave does not measure the airline's quality in terms of service provided to customers under network disturbances, but it measures the airline's richness of alternative routings. Based on the third principal component, we provide the last network indicator NetSize that measures the network size in terms of flight segments or nonstop routes served. We illustrate the position of US carriers at the beginning of our sample (2005) according to the three indicators, NetCenter, NetWeave and NetSize. On this basis, we identify five different network structures ranging from large point-to-point to single-center network. The three indicators propose a network classification different from the traditional one which leads to the usual distinction between legacy carriers and LCCs. This result is consistent with recent analysis on airlines' business models. Empirical studies by Jarach, Zerbini, and Miniero (2009), Klophaus, Conrady, and Fichert (2012) and Bitzan and Peoples (2016) show the convergence in business models between the two categories of operators. We confirm these preliminary observations by analyzing the evolution of the network structures for the US domestic market over a thirteen-year period.

The remainder of this paper is organized as follows. The next section presents a literature review. Section 3 describes the methodology for constructing indicators, combining through a principal component analysis the most important topological measures provided in graph theory. The description of the selected graph theory measures is provided in Section 4. In Section 5 we apply this methodology to the US domestic market at the beginning of our sample, in 2005. We construct three network indicators based on the principal components. A graphical representation of the airline networks through these indicators is provided. Then we built indicators of network evolution that reveal development of an airline network structure over the whole period of observation. Last, we conclude and suggest further possible applications of the indicators.

\section{Literature Review}

We can disentangle two branches within the literature on airline network structures: a graph theory branch with its focus on network mathematical properties and an economic branch that traditionally compares perfect hub-and-spokes and point-to-point networks. In this section, we summarize the main results obtained in each area. In our study, we aim to analyze the airlines' network evolution by linking these two branches. We intend to go beyond the comparison of these two opposite network structures: based on graph theory measures we seek to obtain simple continuous indicators that represent the complex reality of airline network organization.

Air, road and rail transportation are industries organized in networks connecting separate locations. Graph theory provides powerful analytical tools to investigate transportation network structures and their evolution. Several studies in transportation use graph theory to identify network characteristics such as the network robustness to disturbances, the transmission capabilities in the dissemination of information or in spreading communicable diseases, and more generally the evolution of the transport systems. The difficulty arises from the large number of measures available. In general, the literature refers to particular graph theory measures and justifies their relevance for the study of specific objectives. Háznagy et al. (2015) use graph theoretical centrality measures and global characteristics such as network diameter, average path length, degree distribution and community structure to understand and compare network characteristics of public transportation systems in several Hungarian cities. Studies of transportation network resilience can be found in the work of Angeloudis and Fisk (2006) for the world subway system, or Chatterjee, Manohar, and Ramadurai (2016) for bus network in India. Zhu and Luo (2016) calculate average values of degree, clustering coefficient, betweenness or shortest path among others, to characterize Guangzhou's subway network and analyze the evolution of these parameters for the future development of the subway system.

In the airline sector, most of the graph theory studies focus on airport properties. Ryerson and Kim (2013) present a methodology defining tiers for hubs. Grubesic et al. (2008, 2009) evaluate airport hierarchies across time according to passengers flows while Malighetti et al. (2018) study European airport hierarchies according to freight flows. Martín and Voltes-Dorta (2008) propose a hubbing concentration 
index that captures the number of passengers who make some onward connection. ${ }^{5}$ Burghouwt and Redondi (2013) present a compilation of connectivity indicators for airports with most of them coming from graph theory. Other studies analyze different network characteristics at the world, country or regional levels. For instance, Da Rocha (2009), Gautreau, Barrat, and Barthélemy (2009), Wang et al. (2011), Wandelt and Sun (2015), Dunn and Wilkinson (2016) and Du et al. (2016) examine different network properties at the country level. Lordan and Sallan (2017) investigate the robustness of the European airport network in case of airport isolation caused by random or targeted attacks, while Sun, Gollnick, and Wandelt (2017) study the robustness of the worldwide airport network. Diverse applications of the complex network theory to air transportation are summarized in Zanin and Lillo (2013) and discussed in Cook et al. (2015). Few works are dedicated to the network analysis at the airline level. Reggiani et al. (2009a; 2009b) study Lufthansa's network and Cento (2008) analyzes several European carriers' networks.

Although the network structure is a key determinant of airline profitability, costs or delays, the literature is sparse and as stated in the introduction, in most of the cases perfect hub-and-spoke systems are compared to complete point-to-point networks. Hubs are usually reflected in dummies as in Lee and Luengo Prado (2005), Lederman (2008) and Ciliberto and Williams (2010) to study the presence of Hub Premiums in pricing or Fageda and Flores Fillol (2016) to study its effects on congestion. We believe that the absence of continuous network indicators justifies the limitation of the analysis to these two distinct network structures. Some studies, however, have tried to surpass this barrier: Borenstein (1992), Reynolds-Feighan $(1998,2001)$ and Burghouwt, Hakfoort, and Ritsema van Eck (2003) study the airlines hub construction. They use measures such as the Gini index, the Theil entropy measures, the Herfindahl Hirschman Index, the coefficient of variation, or modifications of these measures, depending on the available sources of information, to see whether an airline network has a structure close to a hub-and-spoke. Such measures have been applied to study the impact of hubs on the airline's cost structure (Pels, Nijkamp, and Rietveld 2000; Ryerson and Kim 2014), the level of competition (Hendricks, Piccione, and Tan 1997), prices (Tan and Samuel 2016), the level of congestion and delays (Brueckner 2002, Mayer and Sinai 2003), or to study several of these characteristics combined (Bilotkach, Mueller, and Németh 2014; Brueckner and Zhang 2001).

Nevertheless, hubness is only one network property whereas graph theory measures can characterize other network attributes. One of the challenges faced while applying graph theory is that among many graphtheoretical measures there can be several highly correlated concepts. This complicates the choice of the most appropriate measure and hampers the simultaneous use of similar variables in econometric analysis. Ghobrial (1992) studies the effects of some network characteristics over the aggregated airline's demand and find some "instability" in the estimated coefficients, largely due to the correlation among the network characteristics. For these reasons, we propose to apply a PCA on the most common graph theory measures used in the literature to build tractable indicators. The use of PCA presents two advantages, firstly it avoids choosing a unique graph theory measure to describe individual network structures and secondly it allows combining correlated measures that provide different information about the network characterization. The constructed indicators, that is the focus of our study, will enable in further studies to analyze the economic impact for airlines of their network choices.

\section{Methodology}

The question we address is the following: can we characterize the structure of an airline network so that it could be possible to analyze its evolution, predict airlines' decisions in terms of hub and route creation or evaluate the network optimality? This section presents a methodology to construct network indicators that describe the network structure and its evolution.

\footnotetext{
${ }^{5}$ There exists also a vast literature on hub location that we do not consider here. Alumur and Kara (2008) and Campbell and O'Kelly (2012) propose reviews on hub location models.
} 
The section begins defining airlines' networks with a mathematical concept: graphs. First, we specify the main assumptions to represent networks as graphs. Then we explain the methodological foundation to construct the network indicators.

\subsection{Network Model and Assumptions}

The air transport network is a complex system of flight connections between cities. Such a system can be described by a graph and its structure can be studied using techniques developed in graph theory. A graph is an abstract representation of interconnected objects. It is defined as a set of nodes that are joined by a collection of edges (Diestel 2006). Edges represent relations between the nodes of a graph. We consider the air transport network at the airline level and describe an airline network as a graph. Cities served by the airline are the graph nodes, and flight segments operated between cities are the edges. We study only the network spatial dimension and leave for further research the inclusion of the temporal dimension that is considered by Wojahn (2001) or Burghouwt and de Wit (2005).

In order to apply graph theory tools to study an airline network, a preliminary step devoted to flight data representation is required. The objective of this step is to obtain the data in the form of a connection or adjacency matrix. For an airline, such a matrix describes the existence or absence of an edge between each pair of nodes within the network at a given date. At this stage, we make several assumptions.

Assumption 1: airlines make network decisions at the city level rather than at the airport level.

Although an airline may serve several airports in a metropolitan area, these airports are considered as a single airport for that airline. ${ }^{6}$ Under this assumption, airlines using several airports in the same city take network decisions based on all the flights proposed from all the airports in the city. This is consistent with the airport grouping suggested by Brueckner, Lee, and Singer (2013).

Assumption 2: a graph associated with an airline network is undirected and unweighted. ${ }^{7}$

Our study focuses on structural, or topological, properties of airline networks. This means that we are interested in the network structure in terms of connections between city nodes, no matter the direction, nor any edge characteristics such as flight frequencies or seats. Under assumption 2, we define a flight segment as a dichotomous variable taking value one if a nonstop connection between two cities exists, no matter the direction or the frequencies and taking value zero otherwise. ${ }^{8}$

The last assumption is related to the relationship between major airlines and their feeders. Airlines can decide to operate all the flights they propose to their customers or to outsource the operations to regional carriers or feeder companies. These flights must be coordinated between both carriers.

\section{Assumption 3: major airlines and feeders coordinate their network decisions.}

The choice of subcontracting or integrating routes in a network is crucial in air transport industry as suggested by Forbes and Lederman (2009), Levine (2011) and Gillen, Hasheminia, and Jiang (2015). We assume that network choices for the feeders should be done in perfect coordination with their majors and, therefore, we recode regional/feeder airlines to their major partners. ${ }^{9}$

\footnotetext{
${ }^{6}$ For instance, John F. Kennedy International Airport (JFK) and LaGuardia Airport (LGA) are recoded as NYC for American Airlines as it serves both airports.

7 An edge or a node in a graph can be given a specific quantitative characteristic, or weight. If no weights are assigned to edges or nodes, the graph is said to be unweighted.

${ }^{8}$ The use of undirected graphs implies that nonstop flights between New York and Los Angeles and nonstop flights between Los Angeles and New York are considered as a single flight segment or route. The assumption on undirected graphs is justified for the US domestic market. During the whole period of observation each flight segment has almost the same number of direct and return flights: one-way flights without return equivalent represent $0.54 \%$ of the total number of flights.

${ }^{9}$ For instance, flights operated by Ravn Alaska $(7 \mathrm{H})$ are recoded as Alaska Airlines (AS). This is a common practice in the literature, see for instance Malighetti et al. (2018)
} 


\subsection{Graph Theory and PCA}

Under the previous assumptions an airline network can be represented with an adjacency matrix. If a network evolves over time, its matrix changes accordingly. Each adjacency matrix allows to compute various graph theory measures. In the literature, several of these measures are considered to study the airline networks, however some of them are correlated and in fact may reflect the same network property each providing slightly different information. This complicates the selection of the most appropriate measures and flaws the comparison among existing studies. It is therefore essential to identify the most meaningful variables, or to build some combinations of them, which can reflect accurately the network structure observed from the data. In this article, we propose to follow the latter: we reduce a sample of selected graph theory measures into a small set of indicators built as linear combinations of the measures. The main challenge is then to be able to interpret the indicators in line with the airline network structure.

Reducing the number of variables to a few interpretable linear combinations can be performed by one of the two most widely used dimension-reduction techniques: principal component analysis (PCA) or factor analysis (FA) ${ }^{10}$ Although they share the same objective, to simplify a set of variables, PCA and FA differ analytically. PCA aims to explain most of the total variance observed in the dataset by a smaller set of new components, called principal components. The goal of FA is to understand which factors underlie the covariances between the original variables; it defines the original variables as linear combinations of these factors.

Our purpose is to obtain a reduced set of meaningful variables to describe airline networks and network evolution, through a combination of graph measures, so that the newly constructed variables can effectively identify the network structure and its change over time. Therefore, we focus on the principal component analysis as it allows such transformation. ${ }^{11}$

Once the PCA is applied and a set of principal components is selected, these components must be interpreted with respect to the network structure, so that they can be used in comparing airlines' networks and understanding their evolution. Interpretability of the principal components depends both on the dataset and on the selection of the original variables, this is one of the weaknesses while using PCA. The selection of graph measures to be included in the analysis is crucial to guarantee this interpretability.

We consider various graph theory measures, some of which are commonly used in the transportation literature. A description for each of them will be provided in section 4. For the sake of convenience, we split the graph theory measures into two groups: overall and node-based measures. Overall measures relate to the whole network. In this case a single measure need to be computed for the whole network. We argue that all the overall graph theory measures are robust to changes in the sample and should be used to build airline network indicators with a PCA. Node-based measures assess how nodes (or cities) are related to each other within a network: node-based measures are studied at the node level. Then, in order to obtain measures for the whole network, we need to summarize node measures through centralization indexes and descriptive statistics (maximum, minimum, average, standard deviation, percentiles, etc.). Given this large variety of statistics for node-based measures, their final selection for the implementation of the PCA should be driven by the market characteristics and the possibility to accurately interpret the obtained principal components. Therefore, there is no general rule for the selection of node measures and their contribution to the principal components might vary slightly with sample changes.

\section{Graph Theory Measures}

We now describe all the network measures used in this study and provide their interpretation in terms of airline networks. We distinguish overall network measures that characterize the whole network, from

\footnotetext{
10 See Fodor (2002) for a survey of dimension-reduction techniques.

${ }^{11}$ A detailed description of the technique can be found in Abdi and Williams (2010) and in appendix B for our application to the US domestic market.
} 
node-based network measures that are calculated at the node level and express the relative connectivity of a node compared to the other nodes in the network. Their respective formulas and some illustrative examples are presented in Table A.2.

\subsection{Overall Network Measures}

The elementary network characteristics, i.e., the number of cities (nbCity) and the number of flight segments (nbFS) convey what is called the order of a network and its size, respectively. ${ }^{12}$ The number of flight segments measures the total number of existing nonstop connections between all the cities in the network. The size of the network can be one of the criteria used for network selection: a too small size could lead to the exclusion of a network. The threshold must be determined according to the sample of analyzed networks.

Another concept of network size is the diameter (diamG). It represents, in the case of airlines, the minimum number of nonstop flights needed to connect the two most remote cities in the network. The diameter is another criterion that can be used for network selection. It allows to identify networks with a very small network size as we will see in the next section. The diameter cannot be measured in the case of disconnected networks. As some airlines present disconnected networks, diamG is not included in the PCA. $^{13}$

The network density (DensG), or graph density, is the number of existing edges with respect to all the possible edges. A complete graph, the graph where any pair of nodes has a direct connection, contains all possible connections and thus achieves the maximum density, 1, as can be seen from Table A.2. For any airline network, the higher the value of network density the more nonstop flights the network offers, that is, most of the cities can be reached directly from any city. Typically, a high value of density indicates that the network is close to a point-to-point organization. We argue that this measure must be included into PCA. ${ }^{14}$

\subsection{Node-based Network Measures: Transitivity and Centrality Measures}

Centrality measures are intended to determine the structural relevance of a single node in a network, quantifying in different ways the importance of a node among the other nodes.

The degree centrality of a node (Cdeg) is a purely local measure. It is the number of direct connections the node has with respect to the number of all possible direct connections the node may have. The degree centrality of a node reveals how locally well-connected each node is. In an airline network, the more nonstop flights connect a city with other cities, the higher the degree centrality of this city. For a given network there are as many values of degree centrality as nodes.

A subtler measure of a node's importance is the closeness centrality, which is based on geodesic distance. ${ }^{15}$ However, closeness centrality becomes useless for disconnected graphs since the distance between two nodes belonging to different components is infinite by convention. Da Rocha (2009) extended closeness centrality to disconnected graphs by defining the harmonic centrality index (Char). In an airline network, the harmonic centrality of a city indicates how fast every other city in the network can be reached from this city. A fast trip between two cities means a low number of flight segments required to form the trip. Table A.2 shows that disconnected networks generally have low values of harmonic centrality.

\footnotetext{
12 Note that the network size is measured in terms of segments and not markets. Indeed, airlines can serve markets between two cities via connecting flights; however, that information is not available in our database.

${ }^{13}$ If there are nodes in a graph that cannot be reached from others, the graph is disconnected. In other words, a disconnected graph splits into several connected components, whereas a graph that is in one piece is connected. In Table A.2, all the graphs are connected except in the last two columns which illustrate disconnected graphs.

${ }^{14}$ Given the construction of DensG presented in table A.2, densG, nbCity and nbFS present high correlation levels and can be collinear under some scenarios. To avoid collinearity issues, we decide to exclude nbCity from the PCA.

${ }^{15}$ A geodesic distance between two nodes is the minimum number of non-repeated edges that connect the two nodes, i.e., the number of edges (flights) in a shortest path connecting the nodes (cities).
} 
Betweenness centrality (Cbet) is also derived from geodesics. It quantifies the number of node occurrences on all the graph geodesics. In an airline network, betweenness centrality gives a high value to the city that may occur most often as an intermediate stop on the routes between any pair of other cities containing the least number of stops. In this case a city with a central position will exhibit a high value of betweenness centrality. For instance, the largest value of betweenness centrality is achieved in a star graph by the central node of the star, and equals 1 , whereas all other nodes within the star have betweenness centrality equal to 0 . Note that a high value of betweenness centrality may also refer to cities that play the role of a bridge between two distinct parts of a network as shown in Table A.2 O'Kelly (2016) computes betweenness for nodes among the 30 biggest airlines and compare them to extreme cases as the ones presented in Table A.2.

Eigenvector centrality (Ceig) is based on the concept that connections to high-scoring nodes contribute more to a node score than connections to low-scoring nodes. ${ }^{16}$ A high value of eigenvector centrality for a city characterizes how well this city is connected to other well-connected cities. When a city has a high eigenvector centrality, this may indicate that the city has many direct connections or it is linked to several highly connected cities.

Every centrality measure can be applied to the whole network rather than only to a node, and in that case, we address ourselves to descriptive statistics and centralization indexes. Among the descriptive statistics we can consider the minimum, maximum, average, standard deviation, or different percentiles. The network centralization gives an answer to the question of how central the most central node is in relation to how central all the other nodes are. The centralization indexes belong to [0,1]. Degree, betweenness and harmonic centralization indexes reach their highest value for a star graph. In terms of eigenvector centralization, the most centralized structure is the graph with a single edge (and potentially many isolated nodes). For connected graphs, the value is high if the graph has a single star topology (as illustrated in the first column of Table A.2). Each centralization index is named concatenating ' $G$ ' and the original name of the index, so that the centralization indexes for Cdeg, Char, Cbet, and Ceig become respectively GCdeg, GChar, GCbet, and GCeig.

Finally, a node and a pair of its neighbors ${ }^{17}$ form a triplet that can either be open (when the three nodes are connected by two edges) or closed (when the three nodes are fully connected). The global clustering coefficient, or graph transitivity, is the number of closed triplets over the total number of triplets (both open and closed). The transitivity coefficient (transG) of an airline network can be understood as a measure of the network connectivity. A star network structure has a zero value for transitivity, however as seen in Table A.2 low values of transitivity can also represent other types of structures. Instead, high values of network transitivity, imply the presence of dense groups of nodes. Transitivity is an important measure for the network characterization in air transportation. For instance, Trapote-Barreira, Deutschmann, and Robusté (2016) use network transitivity, associated with network density to study delay propagation.

All these measures represent different network characteristics. As explained previously, some of them, such as the centrality measures, are highly correlated. In order to keep the richness of information provided by these measures we apply PCA to transform them into a few network indicators.

\section{Application to the US Domestic Market}

The methodology proposed in the previous section to construct network indicators is now applied to the case of the US domestic market. Although worldwide data is available in our dataset we choose to focus on this market. The public databases available for the US domestic market will allow to replicate and extend our analysis with complementary information sources such as DB1B database that provides information on

\footnotetext{
${ }^{16}$ The score of a node is proportional to the sum of its neighbors' scores. Hence a node can have a high eigenvector centrality either because it has many neighbors or because its few neighbors are important, or both. A node with high eigenvector centrality will not necessarily be highly connected, and a node with many direct connections will not necessarily have a high eigenvector centrality.

${ }^{17}$ Two nodes are said to be neighbors if they are connected by an edge.
} 
prices. We start this section describing the data source, then we move on to the PCA results, the network characterization and its evolution. Finally, we analyze the robustness of our findings.

\subsection{Data}

Our dataset is built from the Official Airline Guide (OAG), which provides information on worldwide scheduled traffic such as route characteristics (departure and destination airports or distance) and flight characteristics (time of departure and arrival, duration, available seats or operating airlines). We consider one-way direct passenger flights between 2005 and 2018 for all the operating carriers within the US domestic market. Following assumption 1, we group airports belonging to the same city area. Daily data at flight level has been extracted for the third quarter, i.e., July $1^{\text {st }}$ to September $30^{\text {th }}$, since the third quarter presents the highest annual traffic. Collecting the data at daily-flight level is necessary to distinguish marketing and operating carriers for each flight. Following assumption 3, we might treat the operating carrier as a regional/feeder airline and recode it to its major partner, which is the marketing airline. Whenever the operating carrier is different from the marketing carrier, we conduct a detailed analysis of supply at the route level to determine if recoding as legacy is relevant. ${ }^{18}$ Then we aggregate the data at monthly level and finally consider the month of August. The restriction to a single month across years avoids a seasonality treatment that would add no significant information but would be computationally expensive. We replicate the analysis for July and September to show our results' robustness.

The data cleaning process leads to the exclusion of some airlines. We delete from the database non-US operating carriers. These records, representing less than $1 \%$ of the observations, are considered as mistakes since cabotage is forbidden. We exclude small airlines that operate networks with extremely short routes (none exceeding 200 miles) or that exclusively operate aircrafts with a capacity never exceeding 10 seats. In addition, we remove airlines that operate less than 4 years during the period 2005-2018, considering them as non-relevant cases for studying the airlines' network evolution. These two steps in the database cleaning represent together less than $2 \%$ of the observations.

We use the diameter to remove small airlines with a specific network type for which the diameter equals one. This corresponds to 6 airlines. ${ }^{19}$ Half of the airlines in our database have networks that are disconnected. Diameter is not defined for a disconnected graph and consequently this measure is discarded in the subsequent analysis.

The final dataset contains monthly route level information for each operating carrier, for the third quarter of 2005-2018. This represents 157,528 monthly-route observations. The number of operating carriers per year ranges from 14 to $22 .{ }^{20}$ The number of cities varies from 338 to 516 .

\subsection{Three Network Indicators}

The graph measures described in the methodology section are calculated monthly for each operating carrier for the third quarter of 2005-2018. We calculate overall measures and node-based measures. Further, in order to assign node-based measures to the whole network, they are summarized into the usual descriptive statistics and the corresponding centralization indexes are compiled. Therefore, the minimum (min) and maximum (max) over all nodes in the network as well as the mean (mean), median (med), 5th and 95th percentiles (p5 and p95) are computed for degree, harmonic, betweenness and eigenvector centrality measures.

\footnotetext{
${ }^{18} \mathrm{We}$ analyze all the markets where an operator is a marketing carrier for less than $5 \%$ of its flights. Some airlines can operate as feeders for several legacy carriers, in this case, the analysis is done at the market level to properly recode the flights of the operating carrier to the different major partners. A full list of the recoded carriers and markets can be provided upon request.

${ }^{19}$ We eliminated 6 airlines that operate very small networks, with no more than 3 flight segments. Their IATA codes are: 1X, 3E, $\mathrm{CH}, \mathrm{P} 1, \mathrm{U} 5, \mathrm{~V} 2$.

20 The full list of airlines is presented in Appendix A.
} 
However, as discussed in the methodology section, not all the graph measures presented above are equally relevant to describe the network structure and obtain interpretable indicators. We select and include in the PCA the number of flight segments, network density and network transitivity. Regarding degree, harmonic and betweenness, we include their centralization and maximum centrality measures (GCdeg, maxCdeg, GChar, maxChar, GCbet, maxCbet) ${ }^{21}$ This choice is justified as centralization is higher if the network contains very central nodes as well as very peripheral nodes. Star-like networks will have high centralization measures and the most central city will have a high centrality score (i.e., the maximum values of degree, harmonic and betweenness centrality are high). Table A.3 presents the monthly average value and standard deviation for these measures for the period 2005-2018 in August. As can be seen in this table, the betweenness centrality for Frontier Airlines (F9) hub, i.e., Denver, has a value of 0.86 . In point-to-point networks, cities have roughly the same importance within the network, therefore the network will present low centralization measures while the maximum of degree, harmonic and betweenness centrality measures can be high or low.

Finally, we include eigenvector centralization and the mean of eigenvector centrality (GCeig and meanCeig). Eigenvector centrality shows if a city is well-connected to other well-connected cities. Large eigenvector centrality characterizes a city that either has many non-stop connections or one that is connected to many important cities. A high mean value of eigenvector centrality indicates that the cities in the network are on average well connected. Moreover, a value of eigenvector centralization close to zero indicates that most of the cities have roughly the same importance, i.e., all are roughly equally well connected either having the same number of non-stop connections or being connected to important parts of the network, which suggests a point-to-point structure. As we can see in Table A.3, Great Lake Airlines (ZK) network has the highest eigenvector centralization index, while Southwest (WN) has the lowest value. This is consistent with the graph structure of these two airlines: star topology for Great Lake Airlines versus highly connected network for Southwest.

The 11 selected graph-theoretical measures have been calculated for each airline monthly for the third quarter over 14 years: this corresponds to 760 observations on 11 variables. The application of PCA requires stationary variables. Under non-stationary time series, PCA may be difficult to interpret as it can result on few components with similar loadings to all variables, see for instance Lansangan and Barrios (2008). As will be explained in subsection 5.4 our panel data set does not satisfy the stationarity condition. In our case there are two possibilities to obtain stationary data: transform the panel data set into cross sectional data, splitting it by year, or do an analysis based on first differences that could correct for non-stationarity. We combine both approaches by performing first a PCA on the first year of observation, 2005. Through this analysis we can characterize the airline networks. In subsection 5.4 we show that first difference variables satisfy the stationarity condition and allow to build indicators reflecting network evolution. Combining both analysis, we obtain a complete appraisal of the US carriers networks, with first, a characterization of initial structures of the airline networks and second, their subsequent evolution.

The first PCA is performed on the data in 2005 (August), this corresponds to 19 observations on 11 variables. The first three principal components obtained explain $97.85 \%$ of the sample variability. We choose to keep these three principal components corresponding to the largest eigenvalues of 5.5239, 3.2750 and 1.9642 respectively. This choice, as detailed in Appendix B.1, agrees with the commonly used eigenvalue one criterion suggested by F. Kaiser (1960) and with the Cattell's scree test (1966).

The first principal component presents high positive correlations with seven variables (maxCdeg, GCdeg, maxChar, GChar, maxCbet, GCbet, GCeig) as shown in Table 1. These variables correspond to node centrality measures of a network and the respective centralization indexes. Thus, the first principal component can be interpreted as an indicator of the presence of central nodes in the network, or as an indicator of a network topology ranging from point-to-point configuration to a star topology. The larger the value of such an indicator, the closer the network structure to a star; and reversely, low values of the indicator should imply that the network has a point-to-point structure.

\footnotetext{
21 PCA have been tested for several combinations including different sets of node centrality statistics. Our final selection is driven by the possibility to provide an accurate interpretation of the principal components.
} 
Table 1 Correlations between the first three principal components and the original variables; August 2005

\begin{tabular}{rccc}
\hline & PC1 & PC2 & PC3 \\
\hline nbFS & -0.50900 & -0.23337 & 0.81229 \\
densG & -0.00614 & 0.97197 & -0.21223 \\
transG & -0.49323 & 0.83450 & -0.02096 \\
maxCdeg & 0.77331 & 0.49822 & 0.38993 \\
GCdeg & 0.87891 & 0.16974 & 0.44383 \\
maxChar & 0.74777 & 0.48625 & 0.44752 \\
GChar & 0.91378 & -0.05098 & 0.39678 \\
maxCbet & 0.89613 & -0.21353 & -0.36225 \\
GCbet & 0.91237 & -0.22425 & -0.31323 \\
meanCeig & 0.31833 & 0.80097 & -0.47425 \\
GCeig & 0.72118 & -0.57089 & -0.31287 \\
\hline $\begin{array}{r}\text { Percentage of } \\
\text { explained }\end{array}$ & & & \\
variability & & & \\
\hline
\end{tabular}

The second principal component is strongly correlated with three variables. It increases with density (densG), transitivity (transG) and mean eigenvector centrality (meanCeig). This component can be viewed as a measure of network interlacing. A high value of the second principal component may reflect the network richness of alternative routings involving non-stop and one-stop flights, whereas its low value shows that the network contains few or non alternative paths to attain a destination.

The third principal component is strongly correlated with only one of the original variables, flight segments (nbFS). It increases with increasing nbFS, i.e., the number of connections that are offered with nonstop flights. We take this variable in its original form rather than the principal component value and we will denote it as NetSize. ${ }^{22}$

The two first principal components are interpreted as network indicators. Associated with NetSize they allow characterizing the network structure. We denote the first principal component as NetCenter since the component measures the presence of central nodes in an airline network. For the second principal component, the notation NetWeave will be assigned following its interpretation as a measure of network interlacing. We compute the scores on the principal components for each airline in august 2005 . $^{23}$ The way the components are constructed gives rough theoretical limits for the components, each one ranging between -11 and 11(as explained in Appendix B.1). In our dataset, NetCenter ranges from -3.82 to 3.75, and NetWeave, from -1.74 to 5.41. NetSize ranges from 8 to 721 .

\subsection{Airline Network Characterization}

The three indicators constructed in the previous subsection allow comparing the airlines' networks using objective network characteristics.

Based on the airline scores on the three indicators, NetCenter, NetWeave and NetSize, we can characterize the airline networks:

1. An airline network can be classified as a point-to-point network when NetCenter is negative and NetWeave positive; in addition, if NetSize is high, the network is a large point-to-point network; if NetSize is low, the network is a small point-to-point;

\footnotetext{
${ }^{22}$ For instance, Allegiant Air, G4, presents a NetSize of 242 flight segments in 2005 . This means that 242 city pairs are proposed with nonstop flights.

${ }^{23}$ We conducted the same analysis for July and for September 2005. The results are alike, confirming the robustness of the proposed methodology.
} 
2. When NetCenter and NetWeave are both negative, and if NetSize is low, the network has a small multi-center structure;

3. When NetCenter and NetWeave are both negative, and if NetSize is high, the network has a large multi-center structure; in this group some airlines, with lower negative values of NetCenter tends to converge to a point-to-point structure;

4. An airline network can be classified as single-centered when NetCenter is high and NetWeave is close to zero;

5. When NetCenter becomes negative and if NetWeave is negative and low, the network should have a path or a circle structure, however no airline in our database meets this case.

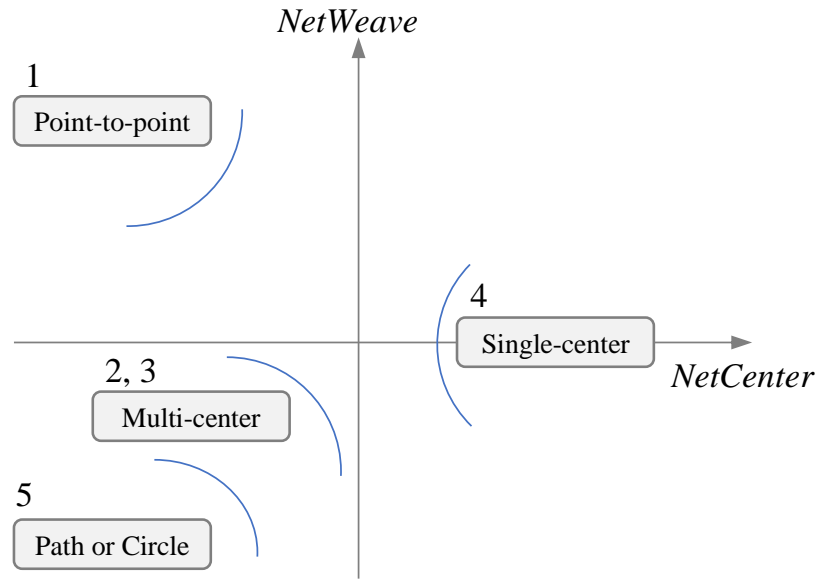

(a)

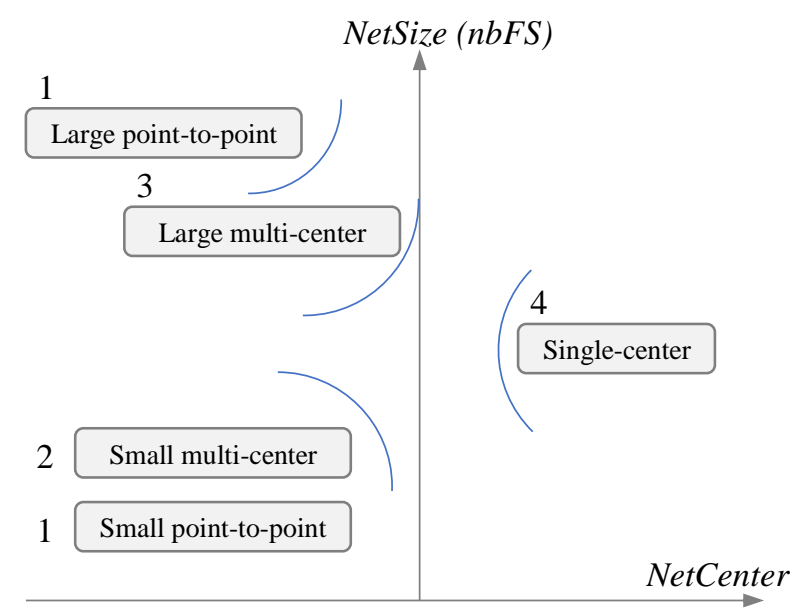

(b)

Figure 1 Network characterization based on NetCenter and NetWeave (a) and on NetCenter and NetSize (b).

Figure 1 displays this classification. The obtained indicators can shed new light on the airline classification between legacy carriers ${ }^{24}$ and low-cost carriers (LCCs). ${ }^{25}$ Figure 2 shows the airlines' positions in August 2005. We observe that LCCs do not exhibit a common pattern in their network structure. Spirit Airlines (NK) and Southwest (WN) have low NetCenter and high NetWeave. This position is characteristic of a point-to-point network. Interestingly, we observe that the other airlines classified into the LCC category are not located in the same area. In August 2005, Frontier Airlines (F9) is a perfect star network (with its hub in Denver) although this airline is nowadays developing several hubs (for instance, Orlando or Las Vegas). JetBlue (B6) has a hub-and-spoke network with several hubs.

Jarach, Zerbini, and Miniero (2009) and Bitzan and Peoples (2016) highlight that both type of airlines could be converging their management strategies towards a hybrid model. Indeed, as seen from NetCenter, there is no clear distinction between legacy carriers and LCCs networks structures. Nonetheless, we observe a difference between the two airline categories in terms of NetWeave. All legacies present negative values for this indicator while most of LCCs present positive values and all LCCs present higher NetWeave values than legacies except Frontier Airlines (F9). This is consistent with the results observed by Lordan and Sallan

\footnotetext{
${ }^{24}$ We include in this category airlines that were established before the Airline Deregulation Act of 1978 except Southwest Airlines, created in 1971, but categorized as LCC: American Airlines (AA), Alaska Airlines (AS), Continental Airlines (CO), Delta Airlines (DL), Hawaiian Airlines (HA), Northwest Airlines (NW), United Airlines (UA) and US Airlines (US). Historically, these airlines provided a higher service level to their passengers than LCCs although the differences have diminished over time. The full list of airlines and their traditional classification is available in Table A.1

${ }^{25}$ LCCs comprise Allegiant Air (G4), JetBlue (B6), Frontier Airline (F9), Spirit Airlines (NK), Virgin America (VX) and Southwest Airlines (WN).
} 
(2017) who study the robustness of airline networks to airport isolation. They find that robustness, their equivalent to NetWeave in our study, is higher for LCCs than for full service carriers over a sample of airlines from Europe, North America and China.

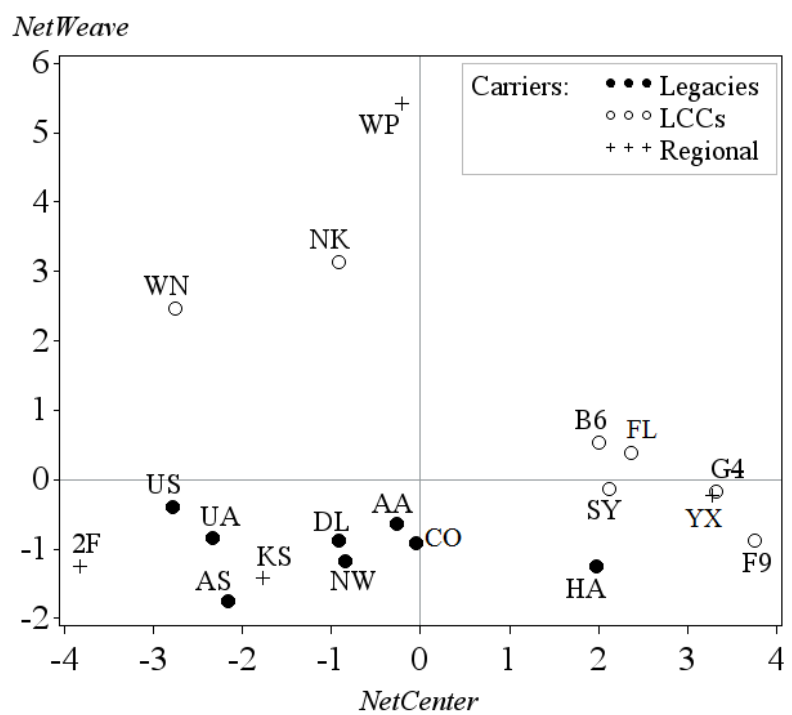

(a)

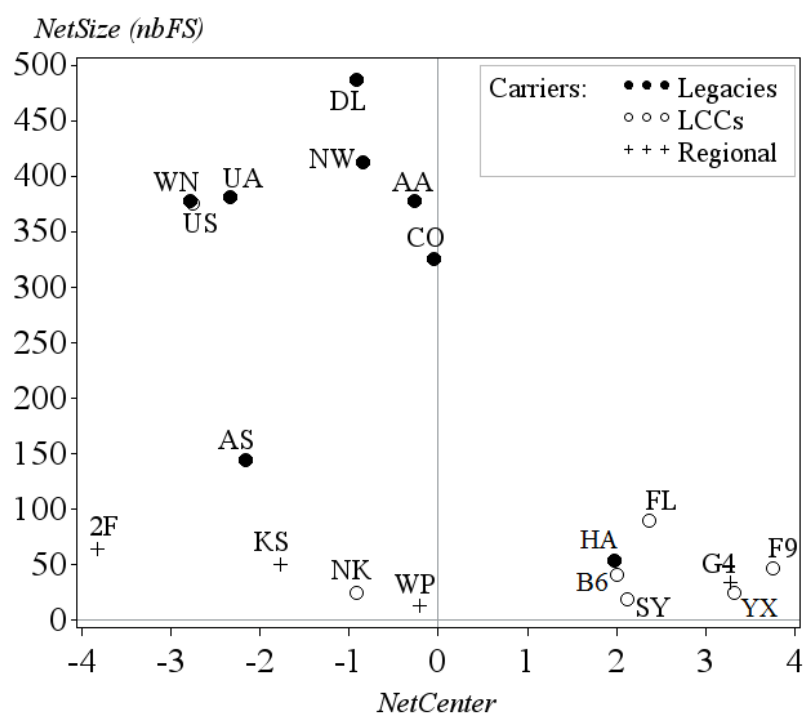

(b)

Figure 2 Airline networks represented in coordinate systems NetCenter-NetWeave (a) and NetCenter-NetSize (b) in August 2005. Points are labeled with airline IATA designators. The full airline list and their respective codes are provided in Table A.1.

Considering the interactions between NetCenter and the third indicator, NetSize, we observe in Figure $2 \mathrm{~b}$ that when NetSize increases, NetCenter decreases to some level between -3 and 0 , both for LCCs and legacies.

Given the relationship between NetCenter, NetWeave and NetSize, an increase in NetSize suggests a tendency toward a multi-center structure. Only Southwest (WN) is apart from this general tendency. Its specific strategy of network expansion will be analyzed in subsection 5.6. The next subsection is dedicated to the construction of indicators allowing to study the network evolution for US domestic carriers over the last decade.

\subsection{Three Network Evolution Indicators}

The extension of the airline network characterization over the whole studied period requires a thorough analysis of the panel data since PCA should be implemented on stationary datasets. As presented in Appendix $\mathrm{C}$ we detected many unit roots among the graph theory measures calculated over the whole period of observation. ${ }^{26}$ Therefore, we use first differences (denoted as $\Delta$ ) to control for non-stationarity and we implement PCA on first difference measures. The derived principal components will be interpreted in terms of evolution.

PCA is performed on the dataset made of the 11 first difference variables of selected graph theory measures calculated for each airline in August from 2005 to 2018; this corresponds to 231 observations for 11 variables. ${ }^{27}$ The first two principal components obtained from the analysis explain $77.86 \%$ of the sample

${ }^{26}$ The unit roots are detected whatever the month considered in the third quarter, July, August or September, for the period from 2005 to 2018.

${ }^{27}$ Although we select one month, August, our results are robust to any change in the selected month within the third quarter. 
variability. We choose to keep these two principal components corresponding to the largest eigenvalues of 5.9041 and 2.6606 respectively. This choice, as detailed in Appendix B.2, agrees with the commonly used eigenvalue one criterion suggested by F. Kaiser (1960). We present in Table 2 the correlations of the first three principal components with the first difference variables. The third principal component is shown in order to demonstrate its noticeably high correlation with the first difference number of flight segments.

Table 2 Correlations between the first three principal components and first differenced variables for 2005-2018

\begin{tabular}{rrr|r}
\multicolumn{4}{c}{ (August) } \\
\hline \multicolumn{1}{c}{ PC1 } & \multicolumn{1}{c}{ PC2 } & \multicolumn{1}{c}{ PC3 } \\
\hline$\Delta$ nbFS & -0.45223 & 0.16266 & 0.83736 \\
$\Delta$ densG & 0.54047 & 0.77873 & -0.03466 \\
$\Delta$ transG & -0.07095 & 0.80756 & -0.21555 \\
$\Delta$ maxCdeg & 0.93891 & 0.16391 & -0.00534 \\
$\Delta$ GCdeg & 0.92110 & -0.11024 & 0.02837 \\
$\Delta$ maxChar & 0.92833 & 0.27665 & 0.11520 \\
$\Delta$ GChar & 0.91426 & -0.19688 & 0.06120 \\
$\Delta$ maxCbet & 0.82104 & -0.37411 & 0.15122 \\
$\Delta$ GCbet & 0.81789 & -0.41597 & 0.15094 \\
$\Delta$ meanCeig & 0.77121 & 0.49835 & -0.01087 \\
$\Delta$ GCeig & 0.19223 & -0.81239 & -0.21396 \\
\hline Percentage of & & & \\
explained & $77.86 \%$ & \\
variability & &
\end{tabular}

$\Delta$ Indicates variables in first differences

The correlations between the first three principal components and the first difference panel variables are similar to the correlations found previously using the initial variables for August 2005. According to the eigenvalue one criterion (F. Kaiser (1960)) and Cattell's scree test (1966), the third principal component is not relevant given its low contribution to the total sample variance. However, given that the first difference in number of flight segments ( $\triangle \mathbf{n b F S}$ ) is highly correlated with PC3, that this variable is the main contributor to this principal component, and that it does not have significant contributions neither to PC1 nor to PC2, we decide to use directly $\Delta \mathbf{n b F S}$ as a third indicator of network evolution renaming it as NetSize $e^{\text {bod }}$. The superscript ${ }^{\text {bod }}$ refers to the fact that the indicators of network evolution are built on first differenced variables.

Using first difference panel variables for PCA, the indicators now represent some increase or decrease in NetCenter, NetWeave and NetSize. Therefore, we interpret the first two principal components as indicators of network evolution in NetCenter and NetWeave. We name the three indicators as NetCenter ${ }^{\text {bod }}$, NetWeave $e^{b o d}$ and NetSize $e^{\text {bod }}$. Their evolution analysis will shed light on the airline individual strategic choice of network expansion as will be shown in subsection 5.6.

\subsection{Robustness}

We have conducted some tests to highlight the robustness of our results. We have applied the same methodology to several randomly selected subsamples: in each draw, we extract $70 \%$ of the observed airlines. In all the cases, F. Kaiser's (1960) eigenvalue one criterion and Cattell's (1966) scree test confirm the selection of our principal components. The correlations of the principal components with the first difference variables remain the same staying their interpretation unchanged.

If the sample selection is not random, PCA results are modified or not and depend on the selection process. Similar results are obtained under constraints such as a sample restriction where minimum frequency levels are imposed. We have restricted the sample with two scenarios: a sample where we observe at least one flight per week and a sample where we observe at least three flights per week. 
Instead, we obtain different results when focusing on big carriers. We build a sample with the 7 biggest carriers, i.e., carriers with more than 300 flight segments per month over the period of analysis. NetSize $e^{\text {bod }}$ is no longer a relevant indicator, which is consistent with the restricted sample where all carriers have a comparable number of flight segments. Only two principal components remain. Although the initial variables' contributions change, the two principal components can be interpreted as NetCenter ${ }^{\text {bod }}$ and NetWeave $e^{\text {bod }}$.

Finally, similar results are obtained when the original variables (not differenced) are used for the PCA over the full period 2005-2018 which confirms the robustness of our results. We argue that the indicators built on differences should be used for the analysis of network evolution as they satisfy the conditions to properly apply a PCA. The following subsection represents such analysis of the airline network evolution through the three network evolution indicators constructed.

\subsection{Representing Airline Network Evolution}

The indicators built on differences, NetCenter ${ }^{\text {bod }}$, NetWeave $e^{\text {bod }}$ and NetSize $e^{\text {bod }}$, and their respective evolutions can be drawn for each airline individually. The figures below present the network expansion of three different airlines, Frontier Airlines (F9), Southwest Airlines (WN), and American Airlines (AA), through the evolution of the three indicators built on differences. For instance, a positive value of NetCenter ${ }^{\text {bod }}$ implies an increase in NetCenter and the magnitude shows the intensity of this change.

We can observe in Figure 3 how Frontier Airlines was developing its network between 2005 and 2018. An increase in route number is reflected in Figure 3 by positive values of NetWeave $e^{b o d}$ and NetSize $e^{b o d}$ during 2013-2016. Negative NetCenter ${ }^{\text {bod }}$ values during this period reflects the development of the network towards a multi-center structure. This is consistent with the airline's expansion strategy as the airline began operations on many routes developing several new hubs since 2013.

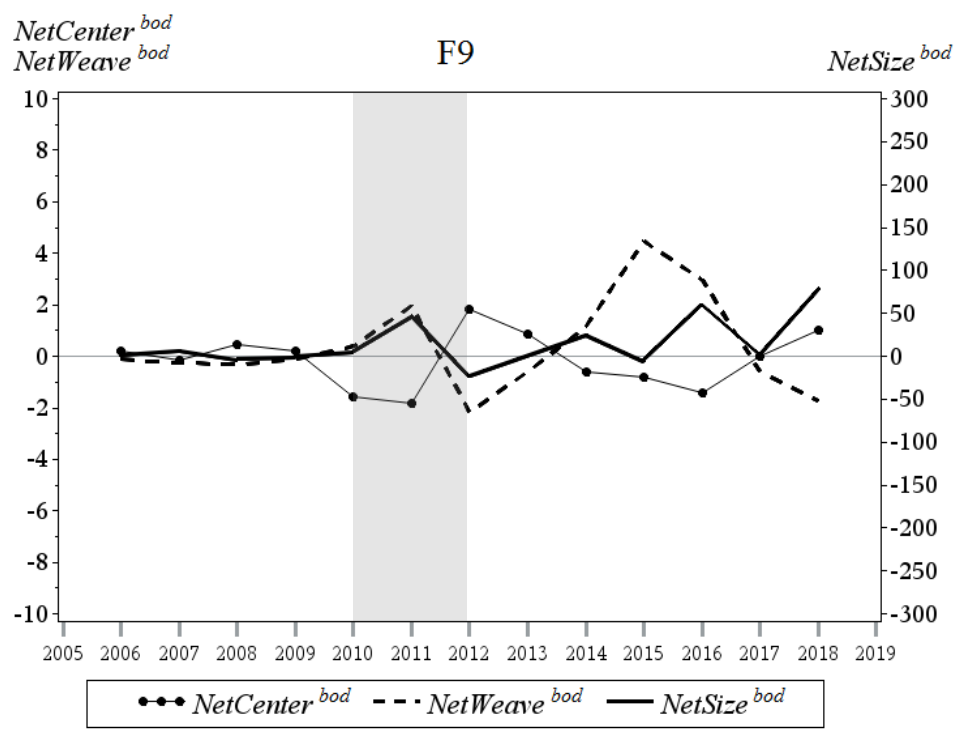

Figure 3 NetCenter ${ }^{b o d}$, NetWeave $e^{\text {bod }}$ and NetSize $e^{\text {bod }}$ evolution for Frontier Airlines. The shadowed area stresses the merger period with Midwest Airlines; from 2011 the indicators characterize the merged network.

Figure 4 presents Southwest Airlines' (WN) network evolution. Southwest, created in 1971, is traditionally quoted in the air transportation literature as the main example of the LCC business model. We can observe the stability of Southwest Airlines' network structure through relatively slight changes in NetCenter ${ }^{\text {bod }}$ and NetWeave $e^{\text {bod }}$ that remain close to zero taking alternatively positive and negative low values. Instead, NetSize $e^{\text {bod }}$ takes positive values on the whole period, but in 2016, reflecting an increase in the number of flight segments. Indeed, Southwest presents a remarkable growth over the studied period: it has 
multiplied by more than 1.5 its number of flight segments between 2005 and 2018. In 2017, Southwest's NetSize was comparable to the post-merger entities of United Airlines (UA) and Continental Airline (CO). Moreover, Southwest's NetWeave indicator is among the highest in our airline sample, and remains stable since 2005. Meanwhile, Southwest's NetCenter indicator remains negative and low. The scores of Southwest's indicators suggest a large network with a point-to-point structure. We therefore highlight an important difference between Southwest's strategy and the other airlines' strategies in terms of network evolution where network expansion is accompanied by a NetWeave reduction.

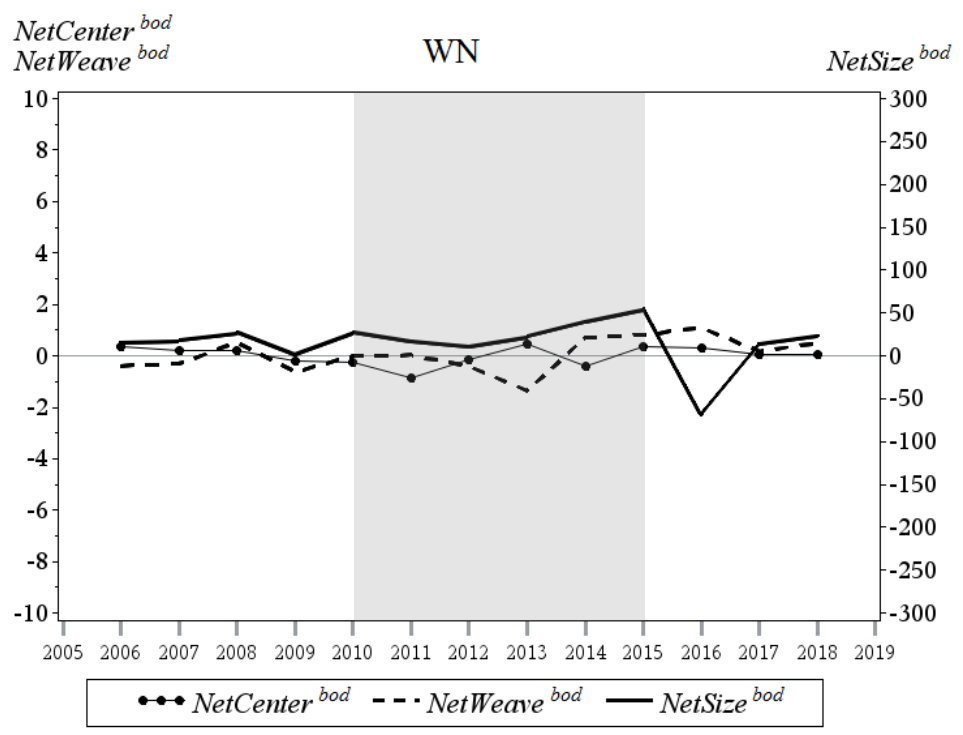

Figure 4 NetCenter ${ }^{b o d}$, NetWeave $e^{\text {bod }}$ and NetSize ${ }^{\text {bod }}$ evolution for Southwest Airlines. The shadowed area stresses the merger period with AirTran Airways; from 2015 the indicators characterize the merged network.

Finally, Figure 5 represents changes in the network evolution indicators for American Airlines. In 2013 the carrier merged with US Airways. The size increase of the carrier's network just after the merger is reflected by high positive value of NetSize $e^{\text {bod }}$. At the same time, we observe positive NetWeave ${ }^{\text {bod }}$ whereas NetCenter ${ }^{\text {bod }}$ is negative, which means that the merging of the two networks was accompanied by overlapping the two route systems and some disorientation of centers in the structure of the resulting network. The carrier rationalizes its network in the post-merger period as reflected by the positive values of NetCenter ${ }^{\text {bod }}$. Becoming the largest airline in the U.S., American Airlines continues to grow in its size: the value of NetSize $e^{\text {bod }}$ remains positive.

The previous example shows how airlines may profoundly modify their networks through mergers. During the considered time frame six mergers took place among the US domestic carriers. Delta Air Lines (DL) and Northwest Airlines (NW) merged between 2008 and 2010. From 2010, these two airlines are considered as one. United Airlines (UA) and Continental Airlines (CO) merged between 2010 and 2012. From 2012, these two airlines are considered as one. Southwest Airlines (WN) acquired AirTran Airways (FL) between 2010 and 2014. From 2015, these two airlines are considered as one. Frontier Airlines (F9) and Midwest Airlines (YX) merged in 2010. From 2011, these two airlines are considered as one. American Airlines (AA) and US Airways (US) merged between 2013 and 2015. From 2016, these two airlines are considered as one. Finally, Alaska Airlines (AS) and Virgin America (VX) merged between 2016 and 2018. From 2018, these two airlines should be considered as one. 


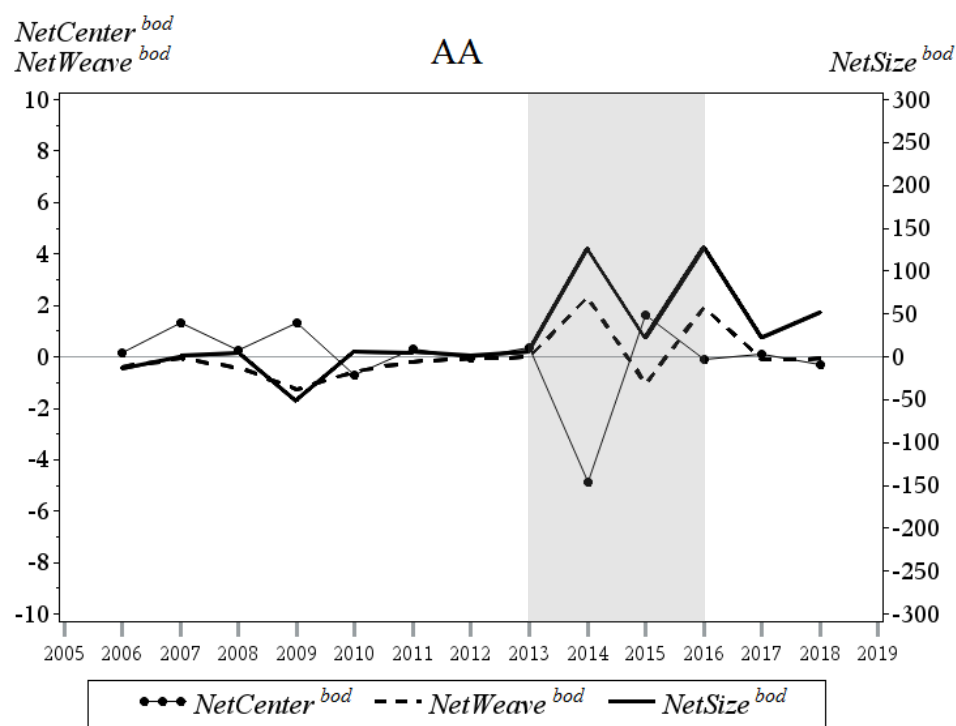

Figure 5 NetCenter ${ }^{b o d}$, NetWeave $e^{\text {bod }}$ and NetSize $e^{b o d}$ evolution for American Airlines. The shadowed area stresses the merger period with US Airways; from 2016 the indicators characterize the merged network.

The impact from mergers can be different depending on the airlines' individual characteristics. For instance, figures 3 and 4 reflect mergers between LCCs. Figure 4 does not reveal a clear impact from the Southwest Airlines merger with AirTran Airways. After the merger, Southwest network characteristics dominate in the merged network as AirTran Airways had a relatively low NetSize compared to Southwest. Instead, a stronger effect can be expected when airlines with similar sizes merge. As shown in Figure 5, the AA-US merger reduced NetCenter ${ }^{\text {bod }}$ while NetWeave ${ }^{\text {bod }}$ and NetSize $e^{\text {bod }}$ were positively affected as the network resulting after this merger exhibited a higher number of hubs. Similar impacts of the mergers are observed in Figure $6(\mathrm{a}, \mathrm{b})$, where we display the DL-NW and UA-CO mergers, respectively, where again the two merging companies were legacies with comparable size before the merger. In both cases, we can clearly observe a significant increase in NetSize just after the merger and a NetCenter decrease. However, from the mergers creation, 2010 for NW and DL, and 2012 for CO and UA, both merged entities move to positive NetCenter ${ }^{\text {bod }}$ values and negative NetSize $e^{\text {bod }}$, certainly due to a network rationalization.

The indicators are therefore able to appraise the impact of mergers over network configurations on a continuous manner. The use of the indicators to measure the impact, instead of a dichotomous variable controlling for mergers shocks, could be advantageous since it allows us to monitor the evolution of the merged entities and measure how the new networks are restructured. 


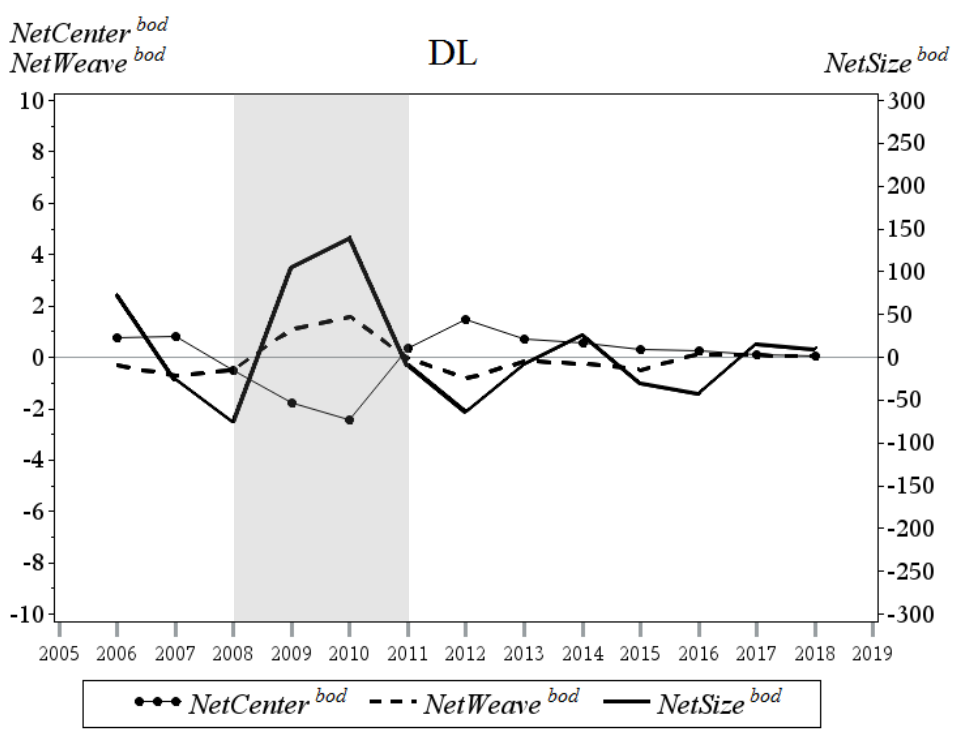

(a)

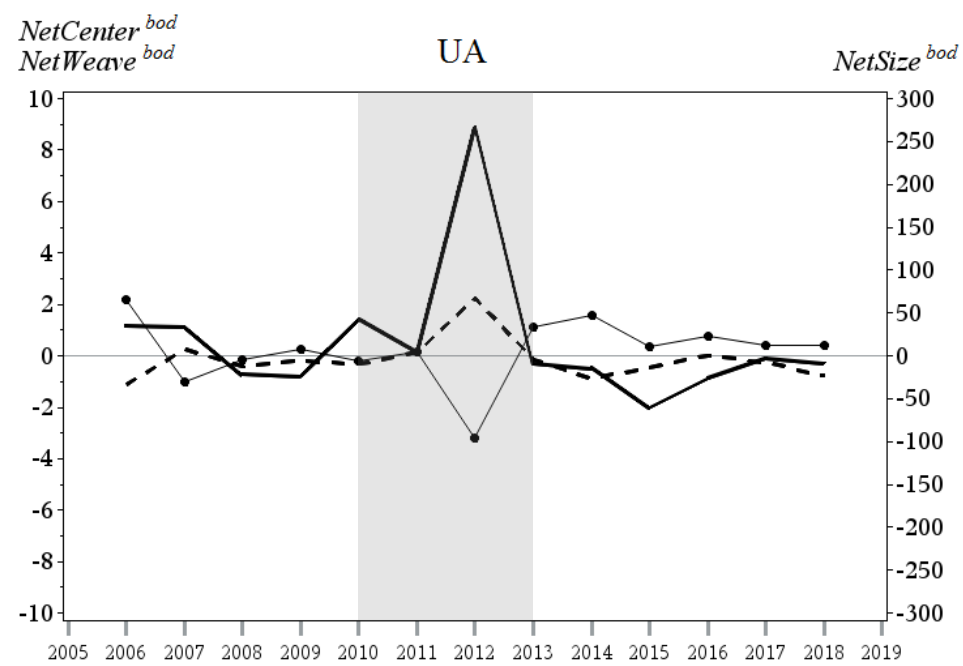

$\bullet \bullet N e t C e n t e r^{\text {bod }}-\cdots$ NetWeave ${ }^{\text {bod }}-$ NetSize $^{\text {bod }}$

(b)

Figure 6 NetCenter ${ }^{\text {bod }}$, NetWeave ${ }^{\text {bod }}$ and NetSize $e^{\text {bod }}$ evolution: (a) for Delta Airlines (merged with Northwest Airlines between 2008 and 2010); from 2010 the indicators characterize the merged network; (b) for United Airlines (merged with Continental Airlines between 2010 and 2012); from

2012 the indicators characterize the merged network. The shadowed areas stress the mergers periods for each carrier.

\section{Conclusion}

The literature review related to airline network analysis revealed the need for some continuous and interpretable instruments to measure airline networks. We aimed to improve the existing studies that oppose hub-and-spoke to fully-connected network when addressing the dependence between network structure and airline economic performances. Some studies propose the use of graph theory measures to characterize a network. The choice of the measure in this case is particularly tricky as the measures are all-complementary to characterize a network. Our objective was to find a method that characterizes an airline network structure through continuous indicators, avoiding the choice of a unique graph measure. To fulfill this objective, we propose to implement the following methodology. First, an airline network should be described as a graph, so that the airline network is represented in the form of a connection, or adjacency, matrix. Such representation must contain information on the existence of nonstop flights provided by each airline between any city pair at a given date. Then, the most relevant set of graph theory measures for the airline industry should be selected and calculated. Lastly, PCA must be performed to reduce the graph theory measures to a small set of new and continuous indicators. These indicators might be interpretable on the basis of relevant choice of graph theory measures. As they are continuous, the indicators can represent the most comprehensive range of network structures. In addition, the methodology proposed in this paper avoids the choice of a unique graph measure to describe the network structure. Instead the indicators combine several measures, each one representing different network properties.

We apply the proposed methodology to the US domestic market and obtain three indicators, NetCenter, NetWeave and NetSize. We provide interpretation for each indicator. NetCenter measures the presence of central airports in a network. The higher NetCenter value, the closer the network is to a star structure that may identify a hub-and-spoke network if connection among flights is ensured. NetWeave indicates the network ability to provide alternative routes. The higher NetWeave, the larger number of one-stop alternative 
paths allowing the airline to join two cities in its network. Finally, NetSize measures the number of flight segments operated by the airline.

According to the three indicators, we can identify five different network structures ranging from large point-to-point to single-center network. This network characterization highlights some differences in terms of network structure among airlines that are not reflected with the traditional distinction between legacies and LCCs. Finally, we illustrate the impact on the indicators of the last main mergers in the US domestic market, Delta - Northwest, United - Continental and American - US Airways. We show that in both cases the airlines see their NetCenter level reduced the year of the merger due to an increase in the number of centers, though afterwards it progressively recovers its initial level. Although this is a known fact, the proposed methodology allows to measure these changes in a continuous manner which enables comparison of the integration level of the networks after a merger.

The methodology provided for characterizing airline networks and measuring their evolution in a simple manner generates new research opportunities. The new network indicators can be used to measure the impact of the network structure on the airlines' cost and profitability, or could be used in assessing the probability to enter a market. These analyses are extremely relevant for the study of airlines strategies. We argue that the obtained indicators should be considered jointly for a more appropriate analysis of these strategies. Regulators will benefit as well from such analyses, with for instance, the evaluation of the impact of networks structures on delays or pollution.

We focus on the US domestic market as information on delays, financial indicators and data on fares are regularly published by the US Department of Transportation for this market. The use of the network indicators associated with these datasets will allow to study the network optimal structure leading to the best airline performances. However, the methodology can be applied to other markets, to different sectors and databases. The indicators can be enhanced using weighted graphs, with weights associated to airports, following the approach of Gautreau, Barrat, and Barthélemy (2009) and Da Rocha (2009). Some weights could also be applied to the flight segments in terms of seats, frequencies, distance or combinations across these variables. The use of weighted graphs increases the difficulty in the calculation of the graph metrics, and is left for further research.

This study focuses on the spatial dimension of the networks which means that we can detect center airports without disentangling hubs from focus cities. One of the study weakness is that flight connectivity, i.e., connections of passengers at hubs, were not covered by the analysis, which would allow such distinction. Further work should consider the temporal dimension as in Wojahn (2001), Burghouwt and de Wit (2005) and Alderighi et al. (2007), that probably would mostly contribute to the first principal component that measures centrality or result into a fourth principal component of PCA. Given that OAG data presents flight information including schedules, potential connectivity among flights can be studied. However, such study requires assumptions on the time needed for connections (minimum and maximum time) and results should be verified with a deep robustness analysis (modifying one or both limits). This is also left for further research. 


\section{Acknowledgements}

We are grateful to Isabelle Laplace (ENAC), Steve Lawford (ENAC), Catherine Muller (TBS), Benny Mantin (University of Waterloo), Sebastien Mitraille (TBS) and participants at the ITEA and ATRS 2017 conferences and at TBS and ENAC research seminars for their valuable advice. We would also like to thank anonymous referees whose comments and suggestions greatly improved the manuscript.

\section{References}

Abdi, Hervé, and Lynne Williams. 2010. "Principal Component Analysis." Wiley Interdisciplinary Reviews: Computational Statistics 2 (July): 433-59. https://doi.org/10.1002/wics.101.

Airbus. 2017. "Global Market Forecast 2017-2036 | Airbus, Commercial Aircraft." Airbus. 2017. http://www.airbus.com/market/global-market-forecast-2017-2036/.

Alderighi, Marco, Alessandro Cento, Peter Nijkamp, and Piet Rietveld. 2005. "Network Competition - the Coexistence of Hub-and-Spoke and Point-to-Point Systems." Journal of Air Transport Management 11 (5): 328-34. https://doi.org/10.1016/j.jairtraman.2005.07.006.

. 2007. "Assessment of New Hub-and-Spoke and Point-to-Point Airline Network Configurations." Transport Reviews 27 (5): 529-549.

Alumur, Sibel, and Bahar Y. Kara. 2008. "Network Hub Location Problems: The State of the Art." European Journal of Operational Research 190 (1): 1-21. https://doi.org/10.1016/j.ejor.2007.06.008.

Angeloudis, Panagiotis, and David Fisk. 2006. "Large Subway Systems as Complex Networks." Physica A: Statistical Mechanics and Its Applications 367 (July): 553-58. https://doi.org/10.1016/j.physa.2005.11.007.

Barla, Philippe, and Christos Constantatos. 2005. "Strategic Interactions and Airline Network Morphology under Demand Uncertainty." European Economic Review 3 (49): 703-16. https://doi.org/10.1016/S0014-2921(03)00054-0.

Bilotkach, Volodymyr, Juergen Mueller, and Adél Németh. 2014. "Estimating the Consumer Welfare Effects of De-Hubbing: The Case of Malév Hungarian Airlines." Transportation Research Part E: Logistics and Transportation Review 66 (Supplement C): 51-65. https://doi.org/10.1016/j.tre.2014.03.001.

Bitzan, John, and James Peoples. 2016. "A Comparative Analysis of Cost Change for Low-Cost, FullService, and Other Carriers in the U.S. Airline Industry." Research in Transportation Economics Forthcoming (December). https://doi.org/10.1016/j.retrec.2016.07.003.

Borenstein, Severin. 1992. "The Evolution of US Airline Competition." The Journal of Economic Perspectives, 1992, 6 (2) edition.

Brueckner, Jan K. 2002. "Airport Congestion When Carriers Have Market Power." The American Economic Review 92 (5): 1357-75.

2004. "Network Structure and Airline Scheduling." The Journal of Industrial Economics 52 (2): 291-312. https://doi.org/10.1111/j.0022-1821.2004.00227.x.

Brueckner, Jan K., Darin Lee, and Ethan Singer. 2013. "City-Pairs Versus Airport-Pairs: A MarketDefinition Methodology for the Airline Industry." Review of Industrial Organization 44 (1): 1-25. https://doi.org/10.1007/s11151-012-9371-7.

Brueckner, Jan K., and Pablo T. Spiller. 1994. "Economies of Traffic Density in the Deregulated Airline Industry." The Journal of Law and Economics 37 (2): 379-415. https://doi.org/10.1086/467318.

Brueckner, Jan K., and Yimin Zhang. 2001. "A Model of Scheduling in Airline Networks: How a Hub-andSpoke System Affects Flight Frequency, Fares and Welfare.” Journal of Transport Economics and Policy 35 (2): 195-222.

Burghouwt, Guillaume, Jacco Hakfoort, and Jan Ritsema van Eck. 2003. "The Spatial Configuration of Airline Networks in Europe." Journal of Air Transport Management, Air Transport Research Society 2003 Special Issue, 9 (5): 309-23. https://doi.org/10.1016/S0969-6997(03)00039-5. 
Burghouwt, Guillaume, and Renato Redondi. 2013. "Connectivity in Air Transport Networks: An Assessment of Models and Applications." Journal of Transport Economics and Policy 47 (January): $35-53$.

Burghouwt, Guillaume, and Jaap de Wit. 2005. "Temporal Configurations of European Airline Networks." Journal of Air Transport Management 11 (3): 185-98. https://doi.org/10.1016/j.jairtraman.2004.08.003.

Campbell, James F., and Morton E. O'Kelly. 2012. "Twenty-Five Years of Hub Location Research." Transportation Science 46 (2): 153-69. https://doi.org/10.1287/trsc.1120.0410.

Cattell, Raymond B. 1966. "The Scree Test For The Number Of Factors.” Multivariate Behavioral Research 1 (2): 245-76. https://doi.org/10.1207/s15327906mbr0102_10.

Caves, Douglas W., Laurits R. Christensen, and Michael W. Tretheway. 1984. "Economies of Density versus Economies of Scale: Why Trunk and Local Service Airline Costs Differ." The RAND Journal of Economics 15 (4): 471-89. https://doi.org/10.2307/2555519.

Cento, Alessandro. 2008. The Airline Industry: Challenges in the 21 st Century. Springer Science \& Business Media.

Chatterjee, Atanu, Manju Manohar, and Gitakrishnan Ramadurai. 2016. "Statistical Analysis of Bus Networks in India." PLOS ONE 11 (12). https://doi.org/10.1371/journal.pone.0168478.

Ciliberto, Federico, and Jonathan W. Williams. 2010. "Limited Access to Airport Facilities and Market Power in the Airline Industry." Journal of Law and Economics 53 (3): 467-495.

Cook, Andrew, Henk A.P. Blom, Fabrizio Lillo, Rosario Nunzio Mantegna, Salvatore Miccichè, Damián Rivas, Rafael Vázquez, and Massimiliano Zanin. 2015. "Applying Complexity Science to Air Traffic Management." Journal of Air Transport Management 42 (January): 149-58. https://doi.org/10.1016/j.jairtraman.2014.09.011.

Da Rocha, Luis EC. 2009. "Structural Evolution of the Brazilian Airport Network." Journal of Statistical Mechanics: Theory and Experiment 2009 (04): P04020.

Diestel, Reinhard. 2006. Graph Theory. Springer Science \& Business Media.

Du, Wen-Bo, Xing-Lian Zhou, Oriol Lordan González, Zhen Wang, Chen Zhao, and Yan-Bo Zhu. 2016. "Analysis of the Chinese Airline Network as Multi-Layer Networks." Transportation Research. Part E, Logistics and Transportation Review 89 (March): 108-16. https://doi.org/10.1016/j.tre.2016.03.009.

Dunn, Sarah, and Sean M. Wilkinson. 2016. "Increasing the Resilience of Air Traffic Networks Using a Network Graph Theory Approach." Transportation Research Part E: Logistics and Transportation Review 90 (June): 39-50. https://doi.org/10.1016/j.tre.2015.09.011.

F. Kaiser, Henry. 1960. "The Application of Electronic Computers to Factor Analysis." Educational and Psychological Measurement 20 (April): 141-51. https://doi.org/10.1177/001316446002000116.

Fageda, Xavier, and Ricardo Flores-Fillol. 2016. "How Do Airlines React to Airport Congestion? The Role of Networks." Regional Science and Urban Economics 56 (January): 73-81. https://doi.org/10.1016/j.regsciurbeco.2015.11.002.

Flores-Fillol, Ricardo. 2009. "Airline Competition and Network Structure." Transportation Research Part B: Methodological 43 (10): 966-83. https://doi.org/10.1016/j.trb.2009.03.002.

Fodor, Imola K. 2002. "A Survey of Dimension Reduction Techniques.” Lawrence Livermore National Lab., CA (US).

Forbes, Silke Januszewski, and Mara Lederman. 2009. "Adaptation and Vertical Integration in the Airline Industry." The American Economic Review 99 (5): 1831-49.

Gautreau, Aurelien, Alain Barrat, and Marc Barthélemy. 2009. "Microdynamics in Stationary Complex Networks." Proceedings of the National Academy of Sciences 106 (22): 8847-8852.

Ghobrial, Atef. 1992. "An Aggregate Demand Model for Domestic Airlines." Journal of Advanced Transportation 26 (2): 105-19. https://doi.org/10.1002/atr.5670260203.

Gillen, David, Hamed Hasheminia, and Changmin Jiang. 2015. "Strategic Considerations behind the Network-Regional Airline Tie Ups - A Theoretical and Empirical Study." Transportation Research Part B: Methodological 72 (Supplement C): 93-111. https://doi.org/10.1016/j.trb.2014.09.001. 
Grubesic, Tony H., Timothy C. Matisziw, and Matthew A. Zook. 2008. "Global Airline Networks and Nodal Regions.” GeoJournal 71 (1): 53-66. https://doi.org/10.1007/s10708-008-9117-0.

2009. "Spatio-Temporal Fluctuations in the Global Airport Hierarchies." Journal of Transport Geography, Airline Industry Liberalization, $17 \quad$ (4): $264-75$. https://doi.org/10.1016/j.jtrangeo.2009.02.003.

Hadri, Kaddour. 2000. "Testing for Stationarity in Heterogeneous Panel Data." The Econometrics Journal 3 (2): 148-61. https://doi.org/10.1111/1368-423X.00043.

Háznagy, Andor, István Fi, András London, and Tamás Nemeth. 2015. "Complex Network Analysis of Public Transportation Networks: A Comprehensive Study.” IEEE. 10.1109/MTITS.2015.7223282.

Hendricks, Ken, Michele Piccione, and Guofu Tan. 1997. "Entry and Exit in Hub-Spoke Networks." RAND Journal of Economics 28 (February): 291-303. https://doi.org/10.2307/2555806.

Jarach, David, Fabrizio Zerbini, and Giulia Miniero. 2009. "When Legacy Carriers Converge with LowCost Carriers: Exploring the Fusion of European Airline Business Models through a Case-Based Analysis." Journal of Air Transport Management - J AIR TRANSP MANAG 15 (November): 28793. https://doi.org/10.1016/j.jairtraman.2009.01.003.

Jolliffe, I.T. 2002. Principal Component Analysis. 2nd ed. New York: Springer-Verlag.

Klophaus, Richard, Roland Dr. Conrady, and Frank Fichert. 2012. "Low Cost Carriers Going Hybrid: Evidence from Europe." Journal of Air Transport Management 23 (August): 54-58. https://doi.org/10.1016/j.jairtraman.2012.01.015.

Lansangan, Joseph Ryan G., and Erniel B. Barrios. 2008. "Principal Components Analysis of Nonstationary Time Series Data.” Statistics and Computing 19 (2): 173. https://doi.org/10.1007/s11222-008-9082y.

Lederman, Mara. 2008. “Are Frequent-Flyer Programs a Cause of the 'Hub Premium'?” Journal of Economics \& Management Strategy 17 (1): 35-66. https://doi.org/10.1111/j.15309134.2008.00170.x.

Lee, Darin, and Maria Jose Luengo-Prado. 2005. "The Impact of Passenger Mix on Reported" Hub Premiums" in the US Airline Industry." Southern Economic Journal, 372-394.

Levin, Andrew, Chien-Fu Lin, and Chia-Shang James Chu. 2002. "Unit Root Tests in Panel Data: Asymptotic and Finite-Sample Properties." Journal of Econometrics 108 (1): 1-24. https://doi.org/10.1016/S0304-4076(01)00098-7.

Levine, Michael. 2011. "Regulation and the Nature of the Firm: The Case of U.S. Regional Airlines." New York University Law and Economics Working Papers, July. http:///sr.nellco.org/nyu_lewp/272.

Lordan, Oriol, and Jose M. Sallan. 2017. "Analyzing the Multilevel Structure of the European Airport Network." Chinese Journal of Aeronautics, April 2017, 30 (2) edition.

Malighetti, Paolo, Gianmaria Martini, Renato Redondi, and Davide Scotti. 2018. “Integrators' Air Transport Networks in Europe." Networks and Spatial Economics, February, 1-25. https://doi.org/10.1007/s11067-018-9390-5.

Martín, Juan Carlos, and Augusto Voltes-Dorta. 2008. "Theoretical Evidence of Existing Pitfalls in Measuring Hubbing Practices in Airline Networks." Networks and Spatial Economics 8 (2-3): 16181. https://doi.org/10.1007/s11067-007-9051-6.

Mayer, Christopher, and Todd Sinai. 2003. "Network Effects, Congestion Externalities, and Air Traffic Delays: Or Why Not All Delays Are Evil.” American Economic Review 93 (February): 1194-1215. https://doi.org/10.1257/000282803769206269.

Nero, Giovanni. 1999. "A Note on the Competitive Advantage of Large Hub-and-Spoke Networks." Transportation Research Part E: Logistics and Transportation Review 35 (4): 225-39. https://doi.org/10.1016/S1366-5545(99)00011-3.

O’Kelly, Morton E. 2016. "Global Airline Networks: Comparative Nodal Access Measures." Spatial Economic Analysis 11 (3): 253-75. https://doi.org/10.1080/17421772.2016.1177262.

Pels, Eric, Peter Nijkamp, and Piet Rietveld. 2000. "A Note on the Optimality of Airline Networks." Economics Letters 69 (3): 429-34. https://doi.org/10.1016/S0165-1765(00)00317-7. 
Pesaran, M. Hashem. 2007. "A Simple Panel Unit Root Test in the Presence of Cross-Section Dependence." Journal of Applied Econometrics 22 (2): 265-312. https://doi.org/10.1002/jae.951.

Reggiani, Aura, Peter Nijkamp, and Alessandro Cento. 2009a. "Connectivity and Competition in Airline Networks." In The Network Experience, 141-63. Springer, Berlin, Heidelberg. https://doi.org/10.1007/978-3-540-85582-8_10.

Reggiani, Aura, Sara Signoretti, Peter Nijkamp, and Alessandro Cento. 2009b. "Network Measures in Civil Air Transport: A Case Study of Lufthansa." In Networks, Topology and Dynamics, 257-82. Lecture Notes in Economics and Mathematical Systems. Springer, Berlin, Heidelberg. https://doi.org/10.1007/978-3-540-68409-1_14.

Reynolds, Aisling. 2001. "Traffic Distribution in Low-Cost and Full-Service Carrier Networks in the US Air Transport Market." Journal of Air Transport Management 7 (September): 265-75. https://doi.org/10.1016/S0969-6997(01)00021-7.

Reynolds-Feighan, Aisling J. 1998. "The Impact of U.S. Airline Deregulation on Airport Traffic Patterns." Geographical Analysis 30 (3): 234-53. https://doi.org/10.1111/j.1538-4632.1998.tb00398.x.

Ryerson, Megan S., and Hyun Kim. 2013. "Integrating Airline Operational Practices into Passenger Airline Hub Definition." Journal of Transport Geography 31 (Supplement C): 84-93. https://doi.org/10.1016/j.jtrangeo.2013.05.013.

- 2014. "The Impact of Airline Mergers and Hub Reorganization on Aviation Fuel Consumption." Journal of Cleaner Production, Special Volume: Making Progress Towards More Sustainable Societies through Lean and Green Initiatives, 85 (Supplement C): 395-407. https://doi.org/10.1016/j.jclepro.2013.12.032.

Silva, Hugo, Erik Verhoef, and Vincent Van den Berg. 2014. "Airline Route Structure Competition and Network Policy." Transportation Research Part B: Methodological 67 (September): 320-343. https://doi.org/10.1016/j.trb.2014.05.012.

Sun, Xiaoqian, Volker Gollnick, and Sebastian Wandelt. 2017. "Robustness Analysis Metrics for Worldwide Airport Network: A Comprehensive Study." Chinese Journal of Aeronautics 30 (2): 500-512. https://doi.org/10.1016/j.cja.2017.01.010.

Tan, Kerry M., and Andrew Samuel. 2016. "The Effect of De-Hubbing on Airfares." Journal of Air $\begin{array}{lllll}\text { Transport } & \text { Management } & 50 & \text { (Supplement } & \text { C): }\end{array}$ https://doi.org/10.1016/j.jairtraman.2015.10.002.

Trapote-Barreira, César, Andreas Deutschmann, and Francesc Robusté. 2016. "Managing Airlines: The Cost of Complexity." Transportation Research Procedia 18: 297-304. https://doi.org/10.1016/j.trpro.2016.12.039.

Wandelt, Sebastian, and Xiaoqian Sun. 2015. "Evolution of the International Air Transportation Country Network from 2002 to 2013." Transportation Research Part E: Logistics and Transportation Review, 2015, 82 (October) edition.

Wang, Jiaoe, Huihui Mo, Fahui Wang, and Fengjun Jin. 2011. "Exploring the Network Structure and Nodal Centrality of China's Air Transport Network: A Complex Network Approach." Journal of Transport Geography 19 (4): 712-21. https://doi.org/10.1016/j.jtrangeo.2010.08.012.

Wojahn, Oliver. 2001. "Airline Network Structure and the Gravity Model." Transportation Research Part E: Logistics and Transportation Review 37 (August): 267-79. https://doi.org/10.1016/S13665545(00)00026-0.

Zanin, Massimiliano, and Fabrizio Lillo. 2013. "Modelling the Air Transport with Complex Networks: A Short Review." European Physical Journal Special Topics 215 (1): 5-21. https://doi.org/10.1140/epjst/e2013-01711-9.

Zhu, Li, and Jinlong Luo. 2016. "The Evolution Analysis of Guangzhou Subway Network by Complex Network Theory." Procedia Engineering, Green Intelligent Transportation System and Safety, 137 (Supplement C): 186-95. https://doi.org/10.1016/j.proeng.2016.01.249. 


\section{Appendix A: Graph measures and descriptive statistics}

Table A.1 Airline name and IATA designator

\begin{tabular}{llc}
\hline \multicolumn{1}{c}{ Airline Name } & IATA Code & Classification \\
\hline Frontier Flying Service & 2F & Regional \\
Gulfstream International Airlines & $3 \mathrm{M}$ before 2011 & Regional \\
Silver Airways Corp & $3 \mathrm{M}$ since 2011 & Regional \\
American Airlines & AA & Legacy \\
Alaska Airlines & AS & Legacy \\
JetBlue Airways & B6 & LCC \\
Continental Airlines & CO & Legacy \\
Delta Air Lines & DL & Legacy \\
Frontier Airlines & F9 & LCC \\
AirTran Airways & FL & LCC \\
Allegiant Air LLC & G4 & LCC \\
Hawaiian Airlines & HA & Legacy \\
PenAir & KS & Regional \\
Spirit Airlines & NK & LCC \\
Northwest Airlines & NW & Legacy \\
Sun Country Airlines & SY & LCC \\
United Airlines & UA & Legacy \\
US Airways & US & Legacy \\
Virgin America & VX & LCC \\
Southwest Airlines & WN & LCC \\
Island Air & WP & Regional \\
Midwest Airlines & YX & Regional \\
Great Lakes Airlines & ZK & Regional \\
\hline
\end{tabular}


Description of notation in Table A.2

\begin{tabular}{|c|c|}
\hline Notation & Description \\
\hline$G$ or $G(V, E)$ & The graph with set of nodes $V$ and edge set $E$. \\
\hline$V$ & The set of nodes of a graph $G(V, E)$. \\
\hline$E$ & The set of edges of a graph $G(V, E)$. \\
\hline$n_{i}$ & An element of $V$, i.e., node $i$ of the graph $G(V, E)$. \\
\hline$e_{i j}$ & An element of $E$, i.e., an edge connecting nodes $n_{i}$ and $n_{j}$. \\
\hline$I_{i}$ & The number of edges between neighbors of node $n_{i}$. \\
\hline$k_{i}$ & The number of neighbors of node $n_{i}$. \\
\hline$c_{X}\left(n_{i}\right)$ & $\begin{array}{l}\text { One of the centrality measures of node } n_{i} \text { in a graph. Subscript } X \text { is assigned to a specific } \\
\text { centrality measure (for instance, degree, } D \text {, or betweenness, } B \text {, etc.) }\end{array}$ \\
\hline$c_{X}\left(n^{*}\right)$ & The largest value of $c_{X}\left(n_{i}\right)$ in a graph; $n^{*}$ is a node that has such value. \\
\hline $\max \sum_{i=1}^{|V|}\left(c_{X}^{*}-c_{X}\left(n_{i}\right)\right)$ & $\begin{array}{l}\text { The maximum possible sum of differences in node centrality } c_{X} \text { for any graph with the } \\
\text { same number of nodes. }\end{array}$ \\
\hline$d\left(n_{i}, n_{j}\right)$ & $\begin{array}{l}\text { The geodesic distance between two nodes } n_{i} \text { and } n_{j} \text {, i.e., the minimum number of non- } \\
\text { repeated edges that connect the two nodes. }\end{array}$ \\
\hline$\underline{g_{j k}(i)}$ & The ratio of the number of geodesics from node $n_{j}$ to node $n_{k}$ passing through node $n_{i}$ to \\
\hline$g_{j k}$ & the total number of geodesics from $n_{j}$ to $n_{k}$. \\
\hline$\lambda$ & A constant. \\
\hline$a_{i j}$ & $\begin{array}{l}\text { An element of the adjacency matrix of a graph } G(V, E) \text {, i.e., of a square matrix } A=\left(a_{i j}\right) \\
\text { with } a_{i j}=1 \text { if nodes } n_{i} \text { and } n_{j} \text { are connected by an edge and } 0 \text { otherwise. }\end{array}$ \\
\hline
\end{tabular}


Table A.2 Examples of graph measures
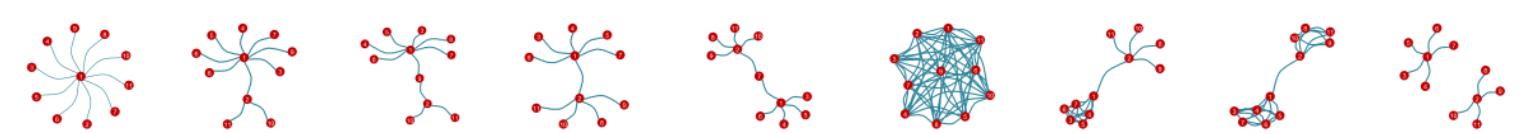

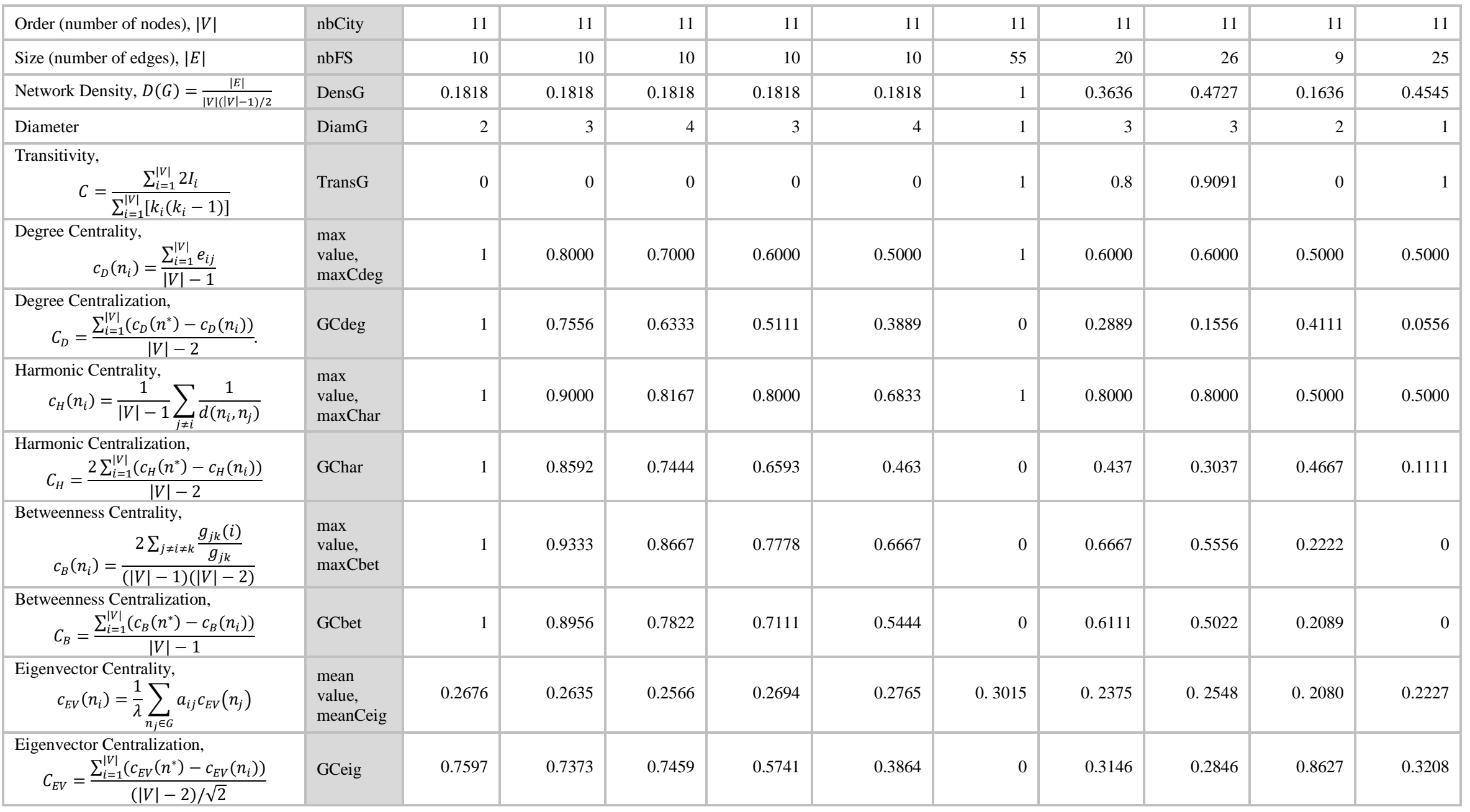


Table A.3 Descriptive statistics: monthly means and standard deviations (in parentheses), in August over 2005-2018

\begin{tabular}{|c|c|c|c|c|c|c|c|c|c|c|c|c|c|c|c|c|c|c|c|c|c|c|c|}
\hline & $2 F$ & $3 M$ & $3 \mathrm{M2}$ & AA & $\mathbf{A S}$ & B6 & $\mathrm{CO}$ & DL & F9 & FL & 4 & HA & KS & NK & NW & SY & UA & US & $V X$ & $N \mathbf{N}$ & NP & YX & K \\
\hline \multirow[t]{2}{*}{ nbCit } & 49 & 12 & 20 & & 132 & 44 & & & . & 1 & & & 28 & 23 & & & & & & & 11 & 81 & 37 \\
\hline & $(24.00)$ & $(2.10)$ & $(9.40)$ & 2.00) & $1.00)$ & $(7.60)$ & $6.00)$ & $6.00)$ & $(9.50)$ & $0.00)$ & $8.00)$ & $(9.20)$ & $90)$ & $.80)$ & (13.00) & $(10.00)$ & $3.00)$ & 7.00) & 1.10) & $.70)$ & $(4.30)$ & $1.00)$ & 7.00) \\
\hline \multirow[t]{2}{*}{ nbAp } & 49 & 12 & 21 & 178 & 134 & 48 & & & ק & 52 & 79 & 19 & 28 & 23 & & 22 & 202 & 165 & 15 & 74 & 11 & 51 & 37 \\
\hline & $(24.00)$ & $(2.10)$ & $(9.00)$ & $(24.00)$ & $0.00)$ & $(9.10)$ & $(27.00)$ & $4.00)$ & (10.00) & (10.00) & $(29.00)$ & $(9.50)$ & $(10.00)$ & $30)$ & $(14.00)$ & $(10.00)$ & $(26.00)$ & (17.00) & $5.00)$ & (9.70) & $(4.30)$ & $2.00)$ & 7.00) \\
\hline \multirow[t]{2}{*}{ nbFs } & 72 & 16 & 28 & 433 & 221 & 100 & 314 & 594 & 98 & 117 & 187 & 26 & 32 & 79 & 374 & 25 & 541 & 419 & 22 & 491 & 14 & 63 & 43 \\
\hline & (41.14) & $(2.22)$ & (11.32) & (130.29) & $(74.81)$ & (23.84) & $(66.50)$ & $0.40)$ & 8.49) & $(31.55)$ & $(112.80)$ & $(9.50)$ & $(14.20)$ & (56.79) & $(35.20)$ & (16.35) & 25.66) & 9.88) & 7.96) & 3.98) & 4.11) & 23.95) & 1.99) \\
\hline \multirow[t]{2}{*}{ dens } & 0.07 & 0.25 & 20 & 03 & 0.03 & 0.11 & 0.03 & 0.03 & 0.06 & 0.09 & 0.06 & 0.20 & 0.10 & 0.28 & .02 & 0.12 & 0.03 & 0.03 & 0.28 & 20 & 0.35 & 0.06 & 0.08 \\
\hline & $(0.04)$ & $(0.06)$ & .14) & $(0.00)$ & $(0.01)$ & $(0.01)$ & $(0.00)$ & $(0.00)$ & $(0.04)$ & $(0.02)$ & $(0.01)$ & $(0.06)$ & $(0.03)$ & $(0.04)$ & $(0.00)$ & $(0.03)$ & $(0.00)$ & $(0.01)$ & $(0.08)$ & $(0.02)$ & $(0.16)$ & $(0.02)$ & (0.05) \\
\hline & 0.20 & 035 & 32 & 0.09 & 010 & 016 & 0.07 & 010 & 0.07 & 0.14 & 0.02 & 0.22 & 0.13 & 0.40 & 0.05 & 0.02 & 0.10 & 0.11 & 0.28 & 0.41 & 0.43 & 0.05 & 0.05 \\
\hline & $(0.02)$ & $(0.03)$ & $(0.08)$ & $(0.03)$ & $(0.02)$ & $(0.03)$ & $(0.01)$ & $(0.02)$ & $(0.10)$ & $(0.04)$ & $(0.02)$ & $(0.09)$ & $(0.04)$ & $(0.10)$ & $(0.01)$ & $(0.03)$ & $(0.02)$ & $(0.02)$ & $(0.06)$ & $(0.05)$ & $(0.08)$ & $(0.01)$ & $0.03)$ \\
\hline \multirow[t]{2}{*}{ maxCdeg } & 0.27 & 0.61 & 0.42 & 0.78 & 0.50 & 0.86 & 0.70 & 0.69 & 0.94 & 0.92 & 0.63 & 0.95 & 0.45 & 0.80 & 0.65 & 0.85 & 0.61 & 0.57 & 0.96 & 0.81 & 0.73 & 0.76 & 0.56 \\
\hline & $(0.11)$ & $(0.27)$ & $(0.10)$ & $(0.00)$ & $(0.16)$ & ) & $(0.10)$ & $(0.07)$ & (0.00) & (0.00) & $(0.12)$ & $(0.14)$ & $(0.17)$ & $(0.08)$ & $(0.04)$ & $(0.14)$ & $(0.05)$ & $(0.06)$ & $(0.06)$ & $(0.04)$ & $(0.27)$ & $(0.21)$ & (0.08) \\
\hline GCdeg & קר? & 0.45 & 25 & 076 & 0.48 & 070 & 068 & 067 & 0.91 & 0.86 & 0.59 & 0.86 & 0.40 & 0.58 & 0.63 & 0.83 & 0.59 & 0.54 & 0.81 & 0.63 & 0.49 & 0.74 & 0.5 \\
\hline \multirow[t]{2}{*}{ maxChar } & 7 & 0.69 & 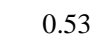 & 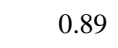 & 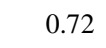 & 0.93 & 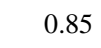 & 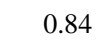 & 0.97 & 0.96 & 0.77 & 0.97 & 0.62 & 0.90 & 0.82 & . & 0.01 & 0.78 & 0.98 & 0.91 & . & 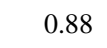 & 0 . \\
\hline & $(0.08)$ & $(0.27)$ & $(0.17)$ & $(0.04)$ & $(0.11)$ & $(0.04)$ & $(0.06)$ & $(0.03)$ & $(0.03)$ & $(0.04)$ & $(0.08)$ & $(0.07)$ & $(0.10)$ & $(0.04)$ & $(0.02)$ & $(0.09)$ & $(0.03)$ & $(0.03)$ & $(0.03)$ & $(0.02)$ & $(0.27)$ & $(0.11)$ & $(0.06)$ \\
\hline \multirow[t]{2}{*}{ GChar } & (15 & 0 & 0.34 & 83 & 060 & 3 & 0.80 & 0 & 0 & 089 & 7 & 088 & 060 & 0 & 07 & 08 & 0 & 068 & 08 & 066 & 0.54 & 08 & 07 \\
\hline & $(0.08)$ & $(0.22)$ & $(0.08)$ & $(0.06)$ & (0.11) & $(0.07)$ & $(0.05)$ & $(0.05)$ & $(0.06)$ & $(0.06)$ & $(0.12)$ & $(0.08)$ & $(0.14)$ & $(0.11)$ & $(0.03)$ & $(0.15)$ & $(0.04)$ & $(0.04)$ & $(0.05)$ & $(0.03)$ & $(0.15)$ & $(0.14)$ & $(0.07)$ \\
\hline \multirow[t]{2}{*}{ maxCbet } & & 0.42 & & 50 & 62 & 0.59 & 0.60 & 0.46 & 0.86 & 070 & 0 & 0 & 0 & 6 & 0.52 & 0 & 0 . & 36 & 0. & 9 & 0.36 & 85 & 80 \\
\hline & $(0.09)$ & $(0.32)$ & $(0.15)$ & $(0.09)$ & $(0.05)$ & $(0.14)$ & $(0.07)$ & $(0.07)$ & $(0.16)$ & $(0.13)$ & $(0.20)$ & $(0.07)$ & $(0.14)$ & $(0.18)$ & $(0.03)$ & $(0.16)$ & $(0.05)$ & $(0.04)$ & $(0.07)$ & $(0.05)$ & $(0.19)$ & $(0.10)$ & $(0.10)$ \\
\hline \multirow[t]{2}{*}{ GCbet } & $06 ?$ & 030 & 2 & 050 & 0 & - & 05 & 04 & 086 & 060 & 0 & 080 & 0 & 0.34 & 0.52 & 0.85 & 0 & 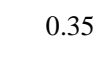 & 0 & 0.18 & 3. & 04 & 0 \\
\hline & $(0.07)$ & $(0.31)$ & $(0.13)$ & $(0.09)$ & $(0.06)$ & $(0.14)$ & $(0.07)$ & $(0.07)$ & $(0.16)$ & $(0.14)$ & $(0.20)$ & $(0.07)$ & $(0.13)$ & $(0.18)$ & $(0.03)$ & $(0.17)$ & $(0.05)$ & $(0.04)$ & $(0.08)$ & $(0.05)$ & $(0.18)$ & $(0.11)$ & $(0.10)$ \\
\hline \multirow[t]{2}{*}{ meanCeig } & 012 & 023 & 018 & م05 & 006 & 012 & 00 & 00 & 01 & 0.11 & 0.1 & ? & 01 & 01 & ק & 01 & (1) & 0.05 & 0 & 0.10 & 027 & 0.12 & 0.14 \\
\hline & $(0.04)$ & $(0.05)$ & $(0.09)$ & $(0.00)$ & $(0.02)$ & $(0.02)$ & $(0.01)$ & $(0.00)$ & $(0.01)$ & $(0.01)$ & $(0.03)$ & $(0.04)$ & $(0.03)$ & $(0.04)$ & $(0.00)$ & $(0.04)$ & $(0.00)$ & $(0.00)$ & $(0.05)$ & $(0.01)$ & $(0.09)$ & $(0.03)$ & $(0.06)$ \\
\hline \multirow[t]{2}{*}{ GCeig } & 0.51 & 0.55 & 0.4 & 0.5 & 0.6 & 0,5 & 0. & 0.4 & 0.74 & 0.61 & 0.55 & 0.58 & 0 & 0.3 & 0.59 & 0.72 & 0.48 & s & 0 & 20 & 48 & 0.75 & 50 \\
\hline & $(0.02)$ & $(0.09)$ & .12) & $0.08)$ & $0.05)$ & $0.04)$ & $(0.02)$ & $(0.06)$ & (0.17) & $(0.08)$ & $(0.12)$ & $(0.09)$ & $(0.14)$ & $(0.10)$ & $(0.02)$ & $(0.10)$ & $(0.05)$ & $(0.04)$ & $(0.03)$ & $(0.03)$ & $(0.15)$ & $(0.07)$ & (0.08) \\
\hline
\end{tabular}




\section{Appendix B: PCA analysis}

PCA constructs principal components as linear combinations of observed variables so that the resulting components are uncorrelated and preserve the maximum amount of the variance of the original data. The weights for the linear combinations are calculated with the constraint that their squares have to sum to 1 (Jolliffe 2002). This is a sequence of constrained maximization problems, such that the first step in the sequence is aimed at finding optimal weights of linear combination for a principal component that accounts for a maximum variance. Each subsequent step gives the next principal component by solving a similar optimization problem with an additional constraint that requires the principal components to be uncorrelated. PCA solves this set of maximization problems, and thus finds all optimal weights for the linear transformation of the original variables to the principal components, due to the eigenvalue decomposition of the correlation matrix. Namely, each principal component is related to an eigenvalue that reflects the variance the component accounts for; the corresponding eigenvector stores the weights of linear combination for the principal component.

\section{B.1: PCA on graph theory variables in 2005}

Table B.1 displays 11 eigenvalues computed on the basis of the correlation matrix for airlines' graph theory measures in 2005. They are ranked in decreasing order according to the amount of variance they account for. Since the correlation matrix provides a standardized measure across all variables, the total variance is equal to the number of variables, i.e., 11. The first component explains most of the variance $(50.22 \%)$, as shows Table B.1. The second component accounts for a maximal amount of variance that is not accounted for by the first component $(29.77 \%)$ and it is uncorrelated with it. Each subsequent component explains less variance until all the variance has been accounted for. The eigenvalues thus add up to the total variance in the data. Since the total variance is equal to the number of variables, the scale of eigenvalues can be interpreted in terms of the number of variables with the highest contribution to the variance.

Table B.1 Eigenvalues of the correlation matrix on original variables in August 2005

\begin{tabular}{rcccc}
\hline & Eigenvalue & Difference & Proportion & Cumulative \\
\hline $\mathbf{1}$ & 5.5239 & 2.2490 & 0.5022 & 0.5022 \\
$\mathbf{2}$ & 3.2750 & 1.3108 & 0.2977 & 0.7999 \\
$\mathbf{3}$ & 1.9642 & 1.8618 & 0.1786 & 0.9785 \\
$\mathbf{4}$ & 0.1024 & 0.0208 & 0.0093 & 0.9878 \\
$\mathbf{5}$ & 0.0816 & 0.0411 & 0.0074 & 0.9952 \\
$\mathbf{6}$ & 0.0405 & 0.0331 & 0.0037 & 0.9989 \\
$\mathbf{7}$ & 0.0074 & 0.0042 & 0.0007 & 0.9995 \\
$\mathbf{8}$ & 0.0032 & 0.0016 & 0.0003 & 0.9998 \\
$\mathbf{9}$ & 0.0016 & 0.0014 & 0.0001 & 1.0000 \\
$\mathbf{1 0}$ & 0.0002 & 0.0001 & 0.0000 & 1.0000 \\
$\mathbf{1 1}$ & 0.0000 & & 0.0000 & 1.0000 \\
\hline
\end{tabular}

The obtained eigenvalues (Table B.1) show that the first three principal components explain $97.85 \%$ of the sample variability. Therefore, these three principal components should be kept as they correspond to the largest eigenvalues of 5.5239, 3.2750 and 1.9642. This choice agrees with commonly used eigenvalue one criterion suggested by F. Kaiser (1960) and Cattell's (1966) scree test. In PCA the total amount of 
variance is equal to the number of variables. Kaiser's criterion shows that the three first eigenvalues obtained correspond to the principal components each capturing most significant amount of data variability greater than one. The remaining components with eigenvalues under 1 are less informative accounting for less variance than is generated by one variable. The scree plot (Figure B.1) confirms the choice. It is a graph of the eigenvalues of all the components. The substantial drop in the magnitude of eigenvalues starts after the third component, thus indicating that the three-component solution would be appropriate to retain for describing the data.
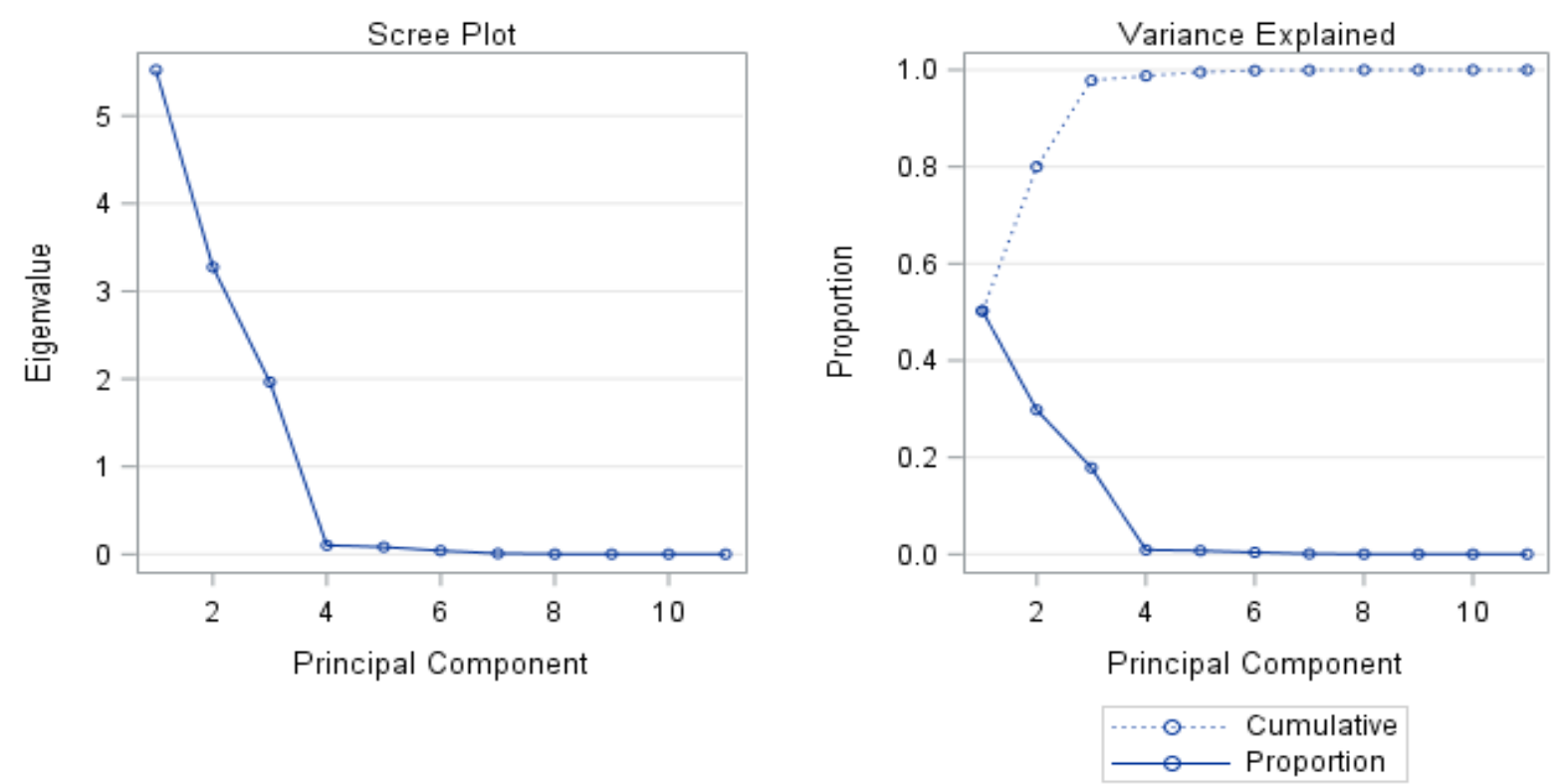

Figure B.1. Scree plot and variance explained by the principal components (PCA on original variables in August 2005).

Each of the eigenvectors presented in Table B.2 is associated with a principal component. This table is the projection matrix that is used to transform the original dataset to a new coordinate system of the principal components.

Table B.2 Eigenvectors (PCA on original variables of August 2005)

\begin{tabular}{rccccccccccc}
\hline & PC1 & PC2 & PC3 & PC4 & PC5 & PC6 & PC7 & PC8 & PC9 & PC10 & PC11 \\
\hline nbFS & -0.2166 & -0.1290 & 0.5796 & -0.1750 & -0.1298 & 0.7385 & -0.0411 & 0.0619 & 0.0266 & 0.0282 & -0.0244 \\
densG & -0.0026 & 0.5371 & -0.1514 & -0.2144 & 0.1299 & 0.2402 & -0.2907 & -0.5729 & -0.2336 & -0.2491 & 0.1967 \\
transG & -0.2099 & 0.4611 & -0.0150 & 0.6823 & 0.3364 & 0.2454 & 0.2488 & 0.1700 & 0.1028 & 0.0540 & 0.0017 \\
maxCdeg & 0.3290 & 0.2753 & 0.2782 & 0.0528 & 0.0230 & -0.0957 & -0.2650 & 0.1341 & -0.5088 & 0.4113 & -0.4580 \\
GCdeg & 0.3740 & 0.0938 & 0.3167 & 0.0375 & -0.0505 & -0.0899 & 0.2465 & 0.3283 & -0.3545 & -0.5182 & 0.4198 \\
maxChar & 0.3182 & 0.2687 & 0.3193 & -0.0439 & 0.0967 & -0.1580 & -0.4264 & 0.1200 & 0.6941 & -0.0369 & 0.0945 \\
GChar & 0.3888 & -0.0282 & 0.2831 & -0.0213 & 0.1186 & -0.0325 & 0.5992 & -0.5636 & 0.1614 & 0.2163 & -0.0523 \\
maxCbet & 0.3813 & -0.1180 & -0.2585 & 0.2762 & -0.3232 & 0.2960 & -0.1660 & -0.0168 & -0.0269 & 0.4495 & 0.5243 \\
GCbet & 0.3882 & -0.1239 & -0.2235 & 0.3012 & -0.2785 & 0.2912 & -0.0673 & -0.0980 & 0.1183 & -0.4790 & -0.5228 \\
meanCeig & 0.1354 & 0.4427 & -0.3384 & -0.4970 & -0.2131 & 0.2025 & 0.3781 & 0.3719 & 0.1580 & 0.1299 & -0.1213 \\
GCeig & 0.3068 & -0.3155 & -0.2232 & -0.1934 & 0.7741 & 0.2827 & -0.0703 & 0.1815 & -0.0456 & -0.0003 & -0.0032 \\
\hline
\end{tabular}


Since PCA was performed based on the correlation matrix, to obtain a value for the principal component, first we need to standardize the raw data; that is, for each variable we need to subtract its mean value and divide the difference by the standard deviation. Then we multiply the row-vector of standardized variables by the eigenvector corresponding to the component. If we denote by $Z_{i}$ a row-vector of standardized data for observation $i$ and by $e_{k}$ the $k$ th eigenvector extracted from PCA, the principal component $P k(i)$ for the observation $i$, is computed as follows:

$$
P k(i)=Z_{i} e_{k}=\left(Z_{i 1}, \ldots, Z_{i 11}\right)\left(\begin{array}{c}
e_{1 k} \\
\vdots \\
e_{11 k}
\end{array}\right)=Z_{i 1} e_{1 k}+\ldots+Z_{i 11} e_{11 k}=\sum_{j=1}^{11} Z_{i j} e_{j k} ;
$$

or using the expression for $Z_{i j}=\frac{X_{i j}-\bar{X}_{j}}{\sigma_{j}}$,

$$
P k(i)=\sum_{j=1}^{11} \frac{X_{i j}-\bar{X}_{j}}{\sigma_{j}},
$$

where for observation $i, X_{i j}$ is the value of $j$ th variable, and $\bar{X}_{j}$ and $\sigma_{j}$ are respectively the mean and standard deviation of the variable.

The number of elements in each vector, $Z_{i}$ and $e_{k}$, is equal to the number of initial variables, i.e., 11 . Since $Z_{i}$ is a vector of standardized data and $e_{k}$ is an eigenvector obtained for the correlation matrix, that in turn contains standardized data, then the maximum absolute value of the vector-product $Z_{i} e_{k}$ is 11 . This means that each principal component $P k(i)=Z_{i} e_{k}$ is between -11 and 11 .

\section{B.2: PCA on difference variables for 2005-2018}

Table B.3 displays the 11 eigenvalues computed on the basis of the correlation matrix for difference variables of airlines' graph theory measures for the period of 2005-2018. The eigenvalues are ranked in decreasing order according to the amount of variance they account for. The total variance is equal to the number of variables, i.e., 11. The eigenvalues thus add up to the total variance in the data. The first component explains most of the variance $(53.67 \%)$. The second component accounts for a maximal amount of variance that is not accounted for by the first component $(24.19 \%)$ and it is uncorrelated with it. Each subsequent component explains less variance until all the variance has been accounted for.

The eigenvalues obtained (Table B.3) show that the first two principal components explain $77.86 \%$ of the sample variability. We choose to keep these two principal components corresponding to the largest eigenvalues of 5.9041 and 2.6606. This choice agrees with eigenvalue one criterion (F. Kaiser 1960) and Cattell's (1966) scree test. Indeed, Kaiser's criterion shows that the two first eigenvalues obtained correspond to the principal components each capturing most significant amount of data variability greater than one. The remaining components with eigenvalues under 1 are less informative accounting for less variance than is generated by one variable. The scree plot (Figure B.2) confirms this choice. The substantial drop in the magnitude of eigenvalues starts after the second component, thus indicating that the twocomponent solution would be appropriate to retain for describing the data. 
Table B.3_ Eigenvalues of the correlation matrix on difference variables for August 2005-2018

\begin{tabular}{rcccc}
\hline & Eigenvalue & Difference & Proportion & Cumulative \\
\hline $\mathbf{1}$ & 5.9041 & 3.2434 & 0.5367 & 0.5367 \\
$\mathbf{2}$ & 2.6606 & 1.8024 & 0.2419 & 0.7786 \\
$\mathbf{3}$ & 0.8582 & 0.2111 & 0.0780 & 0.8566 \\
$\mathbf{4}$ & 0.6472 & 0.2354 & 0.0588 & 0.9155 \\
$\mathbf{5}$ & 0.4117 & 0.0669 & 0.0374 & 0.9529 \\
$\mathbf{6}$ & 0.3449 & 0.2544 & 0.0314 & 0.9842 \\
$\mathbf{7}$ & 0.0905 & 0.0381 & 0.0082 & 0.9925 \\
$\mathbf{8}$ & 0.0523 & 0.0249 & 0.0048 & 0.9972 \\
$\mathbf{9}$ & 0.0274 & 0.0253 & 0.0025 & 0.9997 \\
$\mathbf{1 0}$ & 0.0022 & 0.0013 & 0.0002 & 0.9999 \\
$\mathbf{1 1}$ & 0.0009 & & 0.0001 & 1.0000 \\
\hline
\end{tabular}
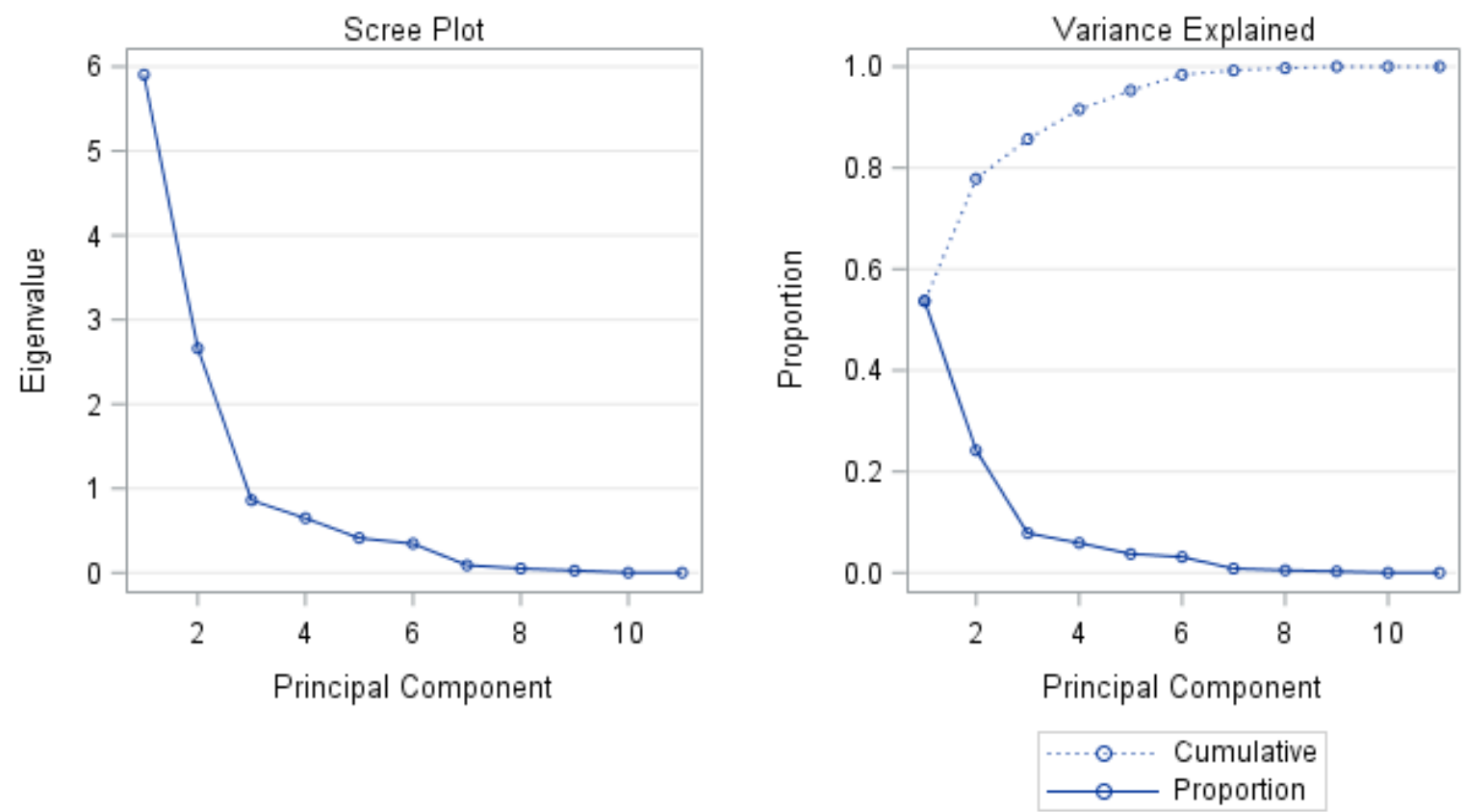

Figure B.2. Scree plot and variance explained by the principal components (PCA on difference variables for 2005-2018).

Each of the eigenvectors presented in Table B.4 is associated with a principal component. This table is the projection matrix that is used to transform the original dataset to a new coordinate system of the principal components. 
Table B.4 Eigenvectors (PCA on difference variables for August 2005-2018)

\begin{tabular}{rccccccccccc}
\hline & PC1 & PC2 & PC3 & PC4 & PC5 & PC6 & PC7 & PC8 & PC9 & PC10 & PC11 \\
\hline$\Delta$ nbFS & -0.1861 & 0.0997 & 0.9039 & 0.2600 & -0.0275 & 0.2625 & 0.0087 & -0.0162 & 0.0199 & -0.0175 & -0.0100 \\
$\Delta$ densG & 0.2224 & 0.4774 & -0.0374 & -0.1717 & -0.2698 & 0.3088 & 0.2909 & 0.1412 & -0.5899 & 0.1410 & 0.2263 \\
$\Delta$ transG & -0.0292 & 0.4951 & -0.2327 & 0.3324 & 0.6718 & 0.3295 & -0.1079 & 0.0360 & 0.1288 & -0.0004 & 0.0017 \\
$\Delta$ maxCdeg & 0.3864 & 0.1005 & -0.0058 & 0.3215 & -0.0574 & -0.1401 & 0.3782 & -0.2213 & -0.0975 & -0.4874 & -0.5245 \\
$\Delta$ GCdeg & 0.3791 & -0.0676 & 0.0306 & 0.4104 & 0.0065 & -0.2116 & 0.0907 & -0.4945 & 0.0579 & 0.3620 & 0.5004 \\
$\Delta$ maxChar & 0.3821 & 0.1696 & 0.1244 & -0.0422 & -0.0443 & -0.1859 & 0.2872 & 0.5987 & 0.5479 & 0.1708 & 0.0351 \\
$\Delta$ GChar & 0.3763 & -0.1207 & 0.0661 & 0.3141 & -0.0042 & -0.1572 & -0.6354 & 0.4153 & -0.3664 & -0.0748 & -0.0074 \\
$\Delta$ maxCbet & 0.3379 & -0.2294 & 0.1632 & -0.3926 & 0.3580 & 0.1656 & 0.0457 & -0.0412 & 0.0176 & -0.5495 & 0.4369 \\
$\Delta$ GCbet & 0.3366 & -0.2550 & 0.1629 & -0.3148 & 0.3858 & 0.1480 & 0.0321 & -0.1141 & -0.1609 & 0.5220 & -0.4613 \\
$\Delta$ meanCeig & 0.3174 & 0.3055 & -0.0117 & -0.2319 & -0.3807 & 0.2981 & -0.4902 & -0.3344 & 0.3825 & -0.0390 & -0.1349 \\
$\Delta$ GCeig & 0.0791 & -0.4980 & -0.2310 & 0.3363 & -0.2188 & 0.6821 & 0.1510 & 0.1658 & 0.1234 & 0.0218 & 0.0090 \\
\hline
\end{tabular}




\section{Appendix C: Tests for Non-Stationarity}

We have implemented several tests for non-stationarity on the graph theory measures selected for the PCA. The first one is the Hadri (2000) Lagrange Multiplier (LM) stationarity test for balanced panel data. We reduce here the sample to airlines observed during the full period 2005-2018 (August). The test sets the null hypothesis that the data are stationary versus the alternative that at least one panel contains a unit root. We specify the robust option to obtain a test robust to heteroskedasticity across panels. We use the demean option to mitigate the impact of cross-sectional dependence, as suggested by Levin, Lin, and Chu (2002). Finally, we include a trend to describe the process by which the series are generated.

Table C. 1 shows that the null assumption is rejected for the initial panel data, although it is not rejected for the fist-difference data. The use of the first differences allows controlling for the presence of unit roots in the initial variables.

Table C. 1 Hadri LM unit root test on balanced panel

\begin{tabular}{lcccc}
\hline & \multicolumn{2}{c}{ Test on initial variables } & \multicolumn{2}{c}{ Test on first difference variables } \\
\cline { 2 - 3 } LM Statistic & p-value & LM Statistic & p-value \\
\hline densG & 8.0195 & 0.0000 & -0.0738 & 0.5294 \\
transG & 3.5315 & 0.0002 & -0.0310 & 0.5124 \\
maxCdeg & 5.6011 & 0.0000 & 0.4918 & 0.3114 \\
GCdeg & 4.8129 & 0.0000 & -0.7156 & 0.7629 \\
maxChar & 5.6657 & 0.0000 & -0.8506 & 0.8025 \\
GCchar & 4.6417 & 0.0000 & -1.0497 & 0.8531 \\
maxCbet & 4.9562 & 0.0000 & -1.0540 & 0.8541 \\
GCbet & 4.4991 & 0.0000 & -1.4217 & 0.9224 \\
meanCeig & 4.4248 & 0.0000 & -1.4353 & 0.9244 \\
GCeig & 1.6276 & 0.0518 & -0.4970 & 0.6904 \\
\hline
\end{tabular}

We have also implemented a second series of tests on the unbalanced panel data. We use the Pesaran (2007) Panel Unit Root test (CIPS) which assumes cross-section dependence. Under the null hypothesis of the CIPS test, the series are AR(1) and therefore non-stationary. The following table shows the results with and without a trend. We cannot reject the null assumption for some of the initial variables when no trend is included in the test, and we cannot reject the null assumption for all the initial variables when we include a trend. We can reject the null assumption for all the first difference variables, whatever the trend option.

Given the results of the unit root tests, we use first difference variables for the Principal Component Analysis. The derived principal components will be interpreted in terms of evolution. 
Table C. 2 Pesaran unit root test on unbalanced panel

\begin{tabular}{|c|c|c|c|c|c|c|c|c|}
\hline & \multicolumn{4}{|c|}{ Specification without trend } & \multicolumn{4}{|c|}{ Specification with trend } \\
\hline & \multicolumn{2}{|c|}{ Initial variable } & \multicolumn{2}{|c|}{ First difference variables } & \multicolumn{2}{|c|}{ Initial variable } & \multicolumn{2}{|c|}{ First difference variables } \\
\hline & Zt-bar & p-value & Zt-bar & p-value & Zt-bar & p-value & Zt-bar & p-value \\
\hline nbFS & -0.754 & 0.225 & -5.029 & 0.000 & 1.071 & 0.858 & -4.073 & 0.000 \\
\hline densG & -3.005 & 0.001 & -6.653 & 0.000 & -0.562 & 0.287 & -2.569 & 0.005 \\
\hline transG & -2.301 & 0.011 & -5.940 & 0.000 & 0.781 & 0.783 & -2.321 & 0.010 \\
\hline $\operatorname{maxCdeg}$ & -2.783 & 0.003 & -5.524 & 0.000 & 0.517 & 0.697 & -1.678 & 0.047 \\
\hline GCdeg & -2.843 & 0.002 & -5.610 & 0.000 & 0.397 & 0.654 & -1.903 & 0.029 \\
\hline maxChar & -2.998 & 0.001 & -5.849 & 0.000 & 0.678 & 0.751 & -2.191 & 0.014 \\
\hline GCchar & -2.065 & 0.019 & -6.436 & 0.000 & 0.390 & 0.652 & -2.317 & 0.010 \\
\hline maxCbet & -2.695 & 0.004 & -6.001 & 0.000 & 0.617 & 0.731 & -2.656 & 0.004 \\
\hline GCbet & -2.744 & 0.003 & -6.101 & 0.000 & 0.511 & 0.695 & -2.714 & 0.003 \\
\hline meanCeig & -1.397 & 0.081 & -5.896 & 0.000 & -0.477 & 0.317 & -2.187 & 0.014 \\
\hline GCeig & -2.498 & 0.006 & -8.949 & 0.000 & -0.711 & 0.239 & -4.641 & 0.000 \\
\hline
\end{tabular}

